\title{
Multiscale analysis of complex aeronautical structures using robust non-intrusive coupling
}

\author{
Stéphane Guinard ${ }^{1}$, Robin Bouclier ${ }^{2}$, Mateus Toniolli ${ }^{3}$ and Jean-Charles Passieux ${ }^{4^{*}}$ (D)
}

\author{
${ }^{*}$ Correspondence: \\ passieux@insa-toulouse.fr \\ ${ }^{4}$ ICA-Université de Toulouse, \\ INSA-UPS-Mines Albi-ISAE-CNRS, \\ Toulouse, France \\ Full list of author information is \\ available at the end of the article
}

\begin{abstract}
The multiscale analysis of large composite aeronautical structures involves the development of robust coupling strategies. Among the latter, non-intrusive coupling is attractive, since it is able to consistently connect a global simplified linear model to a local detailed one, using features available in commercial software. Up to now, such coupling methods were still limited to academic situations where global and local meshes are geometrically and/or topologically conforming and of low geometric complexity. To meet the goal of merging a complex non-planar global shell to a local detailed 3D model, an extension of these techniques is proposed to handle meshes of complex shapes that are not only non-matching but also geometrically and topologically non-conforming. The implemented strategy is original and robust: the innovative nature of the approach is to expand the initial local solid model by generating transitional shell meshing. The generated model incorporates two distinct coupling interfaces: (i) non-intrusive global-local coupling and (ii) shell-solid coupling. The multiscale strategy was successfully validated through different numerical experiments using standard Input/Output of a commercial finite element software. In particular, a representative use-case involving a realistic fuselage section of an aircraft was computed.
\end{abstract}

Keywords: Multiscale computation, Laminated composites, Non-intrusive coupling, Non-conforming geometries, Abaqus v6.14, Co-simulation

\section{Introduction}

During the design, justification and certification process of aircraft structures, design teams have to deal with a number of finite element (FE) computer models. Global models assume simplifications of the geometry, kinematics and behavior: their characteristic size varies between 1 and $10 \mathrm{~m}$, and their main purpose is to estimate loads in large sub-assemblies (fuselage sections, wings, etc.). Local models address greater complexity: their characteristic size varies between 0.1 and $1 \mathrm{~m}$, and their main purpose is to capture the behavior of structural details up to rupture. The ever-increasing power of highperformance computing (HPC [1]) and of design-through-analysis methodologies [2-4] allow the advent of the mythic "complete predictive model": in the long term, local model granularity would be propagated up to large scales, with finally one bulk model driven by smallest scale constraints. This would be irrelevant with today's industrial processes

(c) The Author(s) 2018. This article is distributed under the terms of the Creative Commons Attribution 4.0 International License (http://creativecommons.org/licenses/by/4.0/), which permits unrestricted use, distribution, and reproduction in any medium, provided you give appropriate credit to the original author(s) and the source, provide a link to the Creative Commons license, and indicate if changes were made. 
and engineering mindsets. In particular, aircraft programmes (more broadly, any largescale industrial programme) require permanent access to levels of representation graded in complexity, from the aerodynamic concept to the design of structural details. Consequently, the need to consider models at several scales within a given simulation is, and will continue to be, an essential feature in the development of large sized products of growing complexity.

To ensure interaction between the different scales, it is necessary to construct suitable coupling methods. The most popular method in mechanical engineering is a top-down approach (often known as submodeling [5-8]), which is very present in commercial software. It consists of first carrying out a complete computation of the structure as a whole and then using the solution to prescribe the boundary conditions, as displacement [5] or load [6], on a more refined local model. This method presents the advantage of concentrating the computational effort on the zones that need it most. However, it is mainly reserved for cases in which the local detail has little or no influence on the rest of the structure at global level. The weak point of such a strategy is thus that it is only applied in one direction (from global to local), without considering the repercussions of local, non-linear effects on the global model: this may lead to severe disturbances of the overall balance of the structure $[9,10]$.

For most applications it is necessary to use strong coupling to take the influence of the local model into account beyond its domain of definition. The conventional strategy is then to use monolithic coupling [11-13]. In other words, the coupled solution is obtained through a direct computation (using a single direct solver). This requires a modification of the global model to remove certain elements and replace them by those of the local model. This reorganization of the global mesh (which certainly took many hours, days or even months to be constructed) is extremely intrusive and proves to be incompatible with industrial time cycles.

In response to these problems, a new class of global/local coupling methods, called non-intrusive methods, have recently emerged in the computational structural mechanics community.

Based on an idea put forward by Whitcomb [14] and later formulated by Allix's group [10] for modelling local plasticity, the strategy enables a FE model to be modified locally without having an impact on the corresponding numerical operators. It then becomes easy to interface several software or codes. More precisely, the method relies on an iterative process between global and local computations. The replacement of part of a global model by a more detailed local model can be carried out exactly and non-intrusively: the global model is never modified; only interface displacements and reaction forces are exchanged. This strategy has been successfully applied in many domains (see, e.g., [15, 16] for crack propagation, [17] for taking localized uncertainties into account, [18,19] for non-intrusive plate/3D coupling, [20] for non-linear domain decomposition and [21,22] for transient dynamics analysis). It is of interest to note at this stage that this methodology has some similarities with certain superposition-based multiscale methods that have been proposed long before the concept of non-intrusiveness was introduced (see, e.g, [23] for an overview). These techniques appear in the literature under different names, for example the Chimera method [24], the variational multiscale method [25,26], the FE patches method [27], the numerical zoom [28], the strong coupling method [29], and the $h p-d$ method [30,31]. It should, nevertheless, be stressed that these techniques lead to an 
approximate solution that is expressed as the sum of a global solution and a local correction whereas, in the non-intrusive strategy, the global response is completely eliminated in the zone of interest, its place being taken by the local solution. This limits the interactions between global and local discretizations, which simply become interface integrals, and is the meaning of the term "non-intrusive coupling". The emergence of this type of coupling is thus clearly of major importance as it will enable more accurate sizing and more open exploration of the design envelopes without drastically changing the analysis procedures that are currently established in the industrial community and recognized by the certification authorities.

However, for the time being, the implementation of the method seems to be limited to academic benchmarks, with, for instance, the coupling of geometrically conforming meshes. In other words the global/local interface is aligned with the edges of the local and coarse finite elements, so the local model cannot be truly constructed independently from the global model. This strongly reduces the applicability of the method in an industrial context (see the situation of holes, bolted assemblies, etc.). Recently, the authors proposed a rigorous strategy based on setting up suitable quadrature rules for the coupling of non-conforming NURBS isogeometric models [32,33], but this approach does not seem pertinent in an industrial FE context. To go even further in the non-intrusive philosophy, we propose in the present work to overcome this scientific obstacle by means of a pragmatic technique based on features already implemented in commercial codes.

The target application of this work is the global/local simulation of real composite aeronautical structures. These structures usually involve homogenized non-planar shell models at the global scale and localized full 3D models at the meso-scale, where each ply is meshed to account for local damages like delamination or matrix cracking. To be as general as possible, we consider that the local and global models are neither matching, nor geometrically/topologically conforming. To achieve the goal of simulating local/global coupling on such structures, existing non-intrusive algorithms are not sufficient. A general robust approach is proposed herein, capable to handle all these complexities together. The idea consists in splitting the complexities by using two distinct interfaces: one interface for the local-global coupling, but with conforming meshes, and one interface for the shell/solid coupling. In this respect, the approach is different from [18] where two interfaces where used for the local/global coupling. In addition, the proposed method can treat non-conforming geometries in a more robust and non-intrusive way when compared to [32,33]. The proposed method manages all the numerical complexities that arise with multiscale aeronautical concerns: local non-linear model, non-conforming geometries, shell-to-solid coupling, fully non-planar structure, etc. According to our knowledge, this is the first time, in the context of non-intrusive coupling, that a numerical strategy has been implemented to solve all these inconveniences together.

A demonstrator of the method is developed in the Abaqus v6.14 software in the aim of encouraging its dissemination to the industrial world.

The article is organized as follows: a general presentation of the proposed methodology is made in "The proposed coupling strategy" section. "Implementation" section then gives a more detailed description of the implementation of the strategy in the industrial software Abaqus. "A first validation example" and "Application to industrial realistic examples" sections are devoted to the validation of the approach through numerical experiments. First in "A first validation example" section an academic test case was considered, with 
assumed geometrical simplicity and low numbers of dofs, locally and globally; even if not representative in terms of size and complexity, it does incorporate critical issues to be addressed (non-planar geometry, shell to solid transition, composite material damage and failure). Then in "Application to industrial realistic examples" section the multiscale strategy is exemplified on industrial models derived from actual engineering practices. This allows "Conclusion" section to conclude on the performance of the developed method and on its significant potential for treating concrete engineering problems.

\section{The proposed coupling strategy}

This section introduces the coupling strategy that is implemented for the non-intrusive local enrichment of a shell model with 3D solid elements. After giving a brief overview of the non-intrusive coupling method and highlighting some implementation issues of the current practice, the fundamentals of the proposed technique are described.

\section{Brief overview of non-intrusive coupling}

The non-intrusive coupling strategy seems to be well-established nowadays in the FEbased computational mechanics community (see, e.g., [10,14-22,32-34]).

\section{Reference global/local problem and formulation}

Let us start by considering a global (coarse) model of a structure. The model is characterized by a physical domain $\Omega_{1} \subset \mathbb{R}^{3}$, which is divided into two disjoint, open and bounded subsets $\Omega_{11}$ and $\Omega_{12}$. Those two non-overlapping subdomains share a common interface denoted by $\Gamma$ such that $\Omega_{1}=\Omega_{11} \cup \Omega_{12} \cup \Gamma$ and $\Omega_{11} \cap \Omega_{12}=\varnothing$ (see Fig. 1 (left) for illustration). A simple linear elastic constitutive law is adopted for the global model. We assume that such a behavior is sufficient for the simulation except in the small region $\Omega_{12}$ where a local phenomena is to be introduced. As a consequence, a local (more detailed) "submodel" characterized by domain $\Omega_{2}$ is constructed to replace the global model in $\Omega_{12}$ (see Fig. 1 (right)). The substitution of the local model within the global one is achieved through interface $\Gamma$. The resulting global/local problem to be solved is a classical multi-domain problem in $\Omega_{11} \cup \Omega_{2} \cup \Gamma$, the global solution in $\Omega_{12}$ being discarded (see Fig. 1 (right)). From an engineering point of view, the global model may be thought of as a large-scale finite element model of a macroscopic structure (e.g., an aircraft fuselage). Its construction from CAD data is likely to require important human and computing resources since it may be necessary to make several simplification assumptions to proceed to its meshing. The local model may include any specific local behaviors to allow for a more detailed analysis of the large-scale structure (e.g., local plasticity [10], local fracture [15,16], local uncertainties [17], 3D-shell coupling [18], local contact [20], etc).

Even if the method applies in more general situations, we consider in this section that the local model is linear elastic for simplicity in the presentation. The two non-overlapping subdomains $\Omega_{11}$ and $\Omega_{2}$ are subjected to body forces $\mathbf{f}_{11}^{\mathbf{g}}$ and $\mathbf{f}_{2}^{\mathbf{g}}$, respectively. Furthermore, forces $\mathbf{F}_{11}^{\mathbf{g}}$ and $\mathbf{F}_{2}^{\mathbf{g}}$ are associated to boundaries $\Gamma_{F_{11}}$ and $\Gamma_{F_{2}}$ and, displacements $\mathbf{u}_{11}^{\mathbf{g}}$ and $\mathbf{u}_{2}^{\mathbf{g}}$ are prescribed over boundaries $\Gamma_{u_{11}}$ and $\Gamma_{u_{2}}$ (see Fig. 1 again). The starting point in the derivation of a non-intrusive strategy is to formulate the coupling problem following a Lagrange multiplier approach. Without care of non-intrusiveness at the moment, we write below the classical Lagrange multiplier weak form of the global/local problem. We 


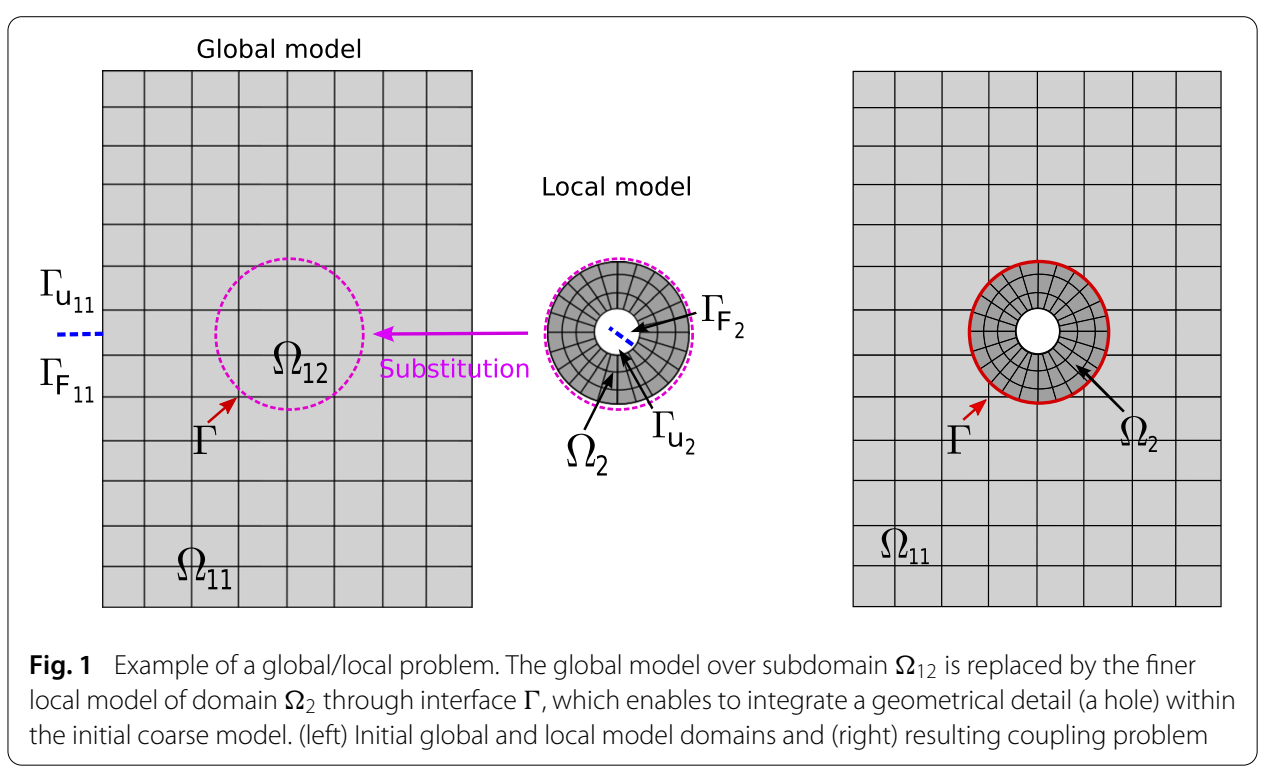

start by defining the functional spaces $\mathcal{U}_{\mathrm{m}}$ and $\mathcal{V}_{m}$ over domain $\Omega_{m}$ (with $m \in\{11,2\}$ ) that will contain the solution and test functions respectively:

$$
\begin{cases}\mathcal{U}_{\mathrm{m}}=\left\{\mathbf{u}_{\mathrm{m}} \in\left[H^{1}\left(\Omega_{m}\right)\right]^{d},\right. & \left.\left.\mathbf{u}_{\mathrm{m}}\right|_{\Gamma_{u_{m}}}=\mathbf{u}_{\mathrm{m}}^{\mathbf{g}}\right\} \\ \mathcal{V}_{m}=\left\{\mathbf{v}_{\mathrm{m}} \in\left[H^{1}\left(\Omega_{m}\right)\right]^{d},\right. & \left.\left.\mathbf{v}_{\mathrm{m}}\right|_{\Gamma_{u_{m}}}=\mathbf{0}\right\} .\end{cases}
$$

A Lagrange multiplier $\lambda \in \mathcal{M}$ (where $\mathcal{M}$ is an appropriate space) is then introduced, as the dual unknown, to impose the coupling constraints across interface $\Gamma$. It results in the following mixed formulation: find $\mathbf{u}_{11} \in \mathcal{U}_{11}, \mathbf{u}_{2} \in \mathcal{U}_{2}$ and $\lambda \in \mathcal{M}$ such that:

$$
\left\{\begin{array}{l}
a_{11}\left(\mathbf{u}_{11}, \mathbf{v}_{11}\right)+b\left(\lambda, \mathbf{v}_{11}\right)=l_{11}\left(\mathbf{v}_{11}\right), \quad \forall \mathbf{v}_{11} \in \mathcal{V}_{11} \\
a_{2}\left(\mathbf{u}_{2}, \mathbf{v}_{2}\right)-b\left(\lambda, \mathbf{v}_{2}\right)=l_{2}\left(\mathbf{v}_{2}\right), \quad \forall \mathbf{v}_{2} \in \mathcal{V}_{2} \\
b\left(\boldsymbol{\mu}, \mathbf{u}_{11}-\mathbf{u}_{2}\right)=0, \quad \forall \boldsymbol{\mu} \in \mathcal{M}
\end{array}\right.
$$

Bilinear form $a_{m}$ and linear form $l_{m}$ associated to domain $\Omega_{m}(m \in\{11,2\})$ read:

$$
\left\{\begin{array}{l}
a_{m}\left(\mathbf{u}_{\mathrm{m}}, \mathbf{v}_{\mathrm{m}}\right)=\int_{\Omega_{m}} \boldsymbol{\varepsilon}\left(\mathbf{v}_{\mathrm{m}}\right): \boldsymbol{C}_{m} \boldsymbol{\varepsilon}\left(\mathbf{u}_{\mathrm{m}}\right) \mathrm{d} \Omega_{m} \\
l_{m}\left(\mathbf{v}_{\mathrm{m}}\right)=\int_{\Omega_{m}} \mathbf{v}_{\mathrm{m}} \cdot \mathbf{f}_{\mathrm{m}}^{\mathbf{g}} \mathrm{d} \Omega_{m}+\int_{\Gamma_{F_{m}}} \mathbf{v}_{\mathrm{m}} \cdot \mathbf{F}_{\mathrm{m}}^{\mathbf{g}} \mathrm{d} \Gamma_{F_{m}} ;
\end{array}\right.
$$

where $\boldsymbol{\varepsilon}$ denotes the infinitesimal strain tensor and $\boldsymbol{C}_{\mathrm{m}}$ the Hooke tensors. Finally, bilinear form $b$ is defined such that:

$$
b(\boldsymbol{\mu}, \mathbf{u})=\int_{\Gamma} \boldsymbol{\mu} \cdot \mathbf{u} \mathrm{d} \Gamma .
$$

We now formulate the problem in the discrete setting. To this end, we introduce the finite element shape functions $\left(N_{A}^{1}\right)_{A \in\left\{1,2, \ldots, n_{1}\right\}}$ and $\left(N_{B}^{2}\right)_{B \in\left\{1,2, \ldots, n_{2}\right\}}$ that discretize the global and local model, respectively. In addition, we denote by $\left(N_{C}^{11}\right)_{C \in\left\{1,2, \ldots, n_{11}\right\}}$ the restricted part to domain $\Omega_{11}$ of the basis functions of the global model. Following the 
principle of isoparametric elements, the basis $\left(N_{C}^{11}\right)_{C \in\left\{1,2, \ldots, n_{11}\right\}}$ and $\left(N_{B}^{2}\right)_{B \in\left\{1,2, \ldots, n_{2}\right\}}$ are used to build the finite element spaces $\mathcal{U}_{11}^{h}$ and $\mathcal{U}_{2}^{h}$ corresponding to the discretization of $\mathcal{U}_{11}$ and $\mathcal{U}_{2}$, respectively. Finally, the finite element space for the Lagrange multiplier is denoted by $\mathcal{M}^{h}$ and the corresponding basis functions read $\left(N_{D}^{\lambda}\right)_{D \in\left\{1,2, \ldots, n_{\lambda}\right\}}$. The construction of space $\mathcal{M}^{h}$ may require special attention to avoid numerical problems. At this stage, we do not give more information regarding this point. This will be discussed further in the paper. By substituting the finite element approximations of $\mathbf{u}_{11}, \mathbf{u}_{2}$ and $\lambda$ in the weak form (2), and denoting the associated degrees of freedom vectors by $\left\{U_{11}\right\},\left\{U_{2}\right\}$ and $\{\Lambda\}$, the following linear system is obtained:

$$
\left[\begin{array}{ccc}
{\left[K_{11}\right]} & {[0]} & {\left[C_{11}\right]^{T}} \\
{[0]} & {\left[K_{2}\right]} & -\left[C_{2}\right]^{T} \\
{\left[C_{11}\right]-\left[C_{2}\right]} & {[0]}
\end{array}\right]\left\{\begin{array}{c}
\left\{U_{11}\right\} \\
\left\{U_{2}\right\} \\
\{\Lambda\}
\end{array}\right\}=\left\{\begin{array}{c}
\left\{F_{11}\right\} \\
\left\{F_{2}\right\} \\
\{0\}
\end{array}\right\} .
$$

Operators $\left[K_{11}\right]$ (respectively $\left.\left\{F_{11}\right\}\right)$ and $\left[K_{2}\right]$ (resp. $\left\{F_{2}\right\}$ ) are the classical stiffness matrices (resp. vector forces) associated to domains $\Omega_{11}$ and $\Omega_{2}$. $\left[C_{11}\right]$ and $\left[C_{2}\right]$ are the mortar coupling operators that formally reads as follows:

$$
\left[C_{11}\right]=\int_{\Gamma}\left[N_{\lambda}\right]^{T}\left[N_{11}\right] \mathrm{d} \Gamma ; \quad\left[C_{2}\right]=\int_{\Gamma}\left[N_{\lambda}\right]^{T}\left[N_{2}\right] \mathrm{d} \Gamma
$$

where $\left[N_{11}\right]$ (resp. $\left[N_{2}\right]$ and $\left[N_{\lambda}\right]$ ) represents the standard shape function matrix related to subspace $\mathcal{U}_{11}^{h}$ (resp. $\mathcal{U}_{2}^{h}$ and $\left.\mathcal{M}^{h}\right)$.

Resolution (5) of the global/local problem constitutes the classical monolithic approach: the resulting model of Fig. 1 (right) is computed directly using a single direct solver. This method is intrusive because the global model is cut to remove some of its elements (and possibly pieces of elements), that are replaced by the elements of the local model (see Fig. 1 again). It is thus necessary to modify a complex large-scale finite element model that may have already required an important time to be established, which is not convenient in an industrial context. In case the local detail grows up [16,20], it is even more costly since the global operator must be re-assembled and refactorized each time the local model evolves. Therefore, a very desirable feature is the non-modification of the global model (and associated finite element operators) built by industry. This is the main aspect of the non-intrusive coupling resolution that is introduced in the following.

\section{Iterative non-intrusive resolution}

Rather than directly solving the system of Eq. (5), the non-intrusive strategy is based on an iterative exchange procedure. We first split the initial system (5) in order to identify a Neumann problem over $\Omega_{11}$ and a Dirichlet problem over $\Omega_{2}$. Then, in order to involve the contributions of the global model over the whole domain $\Omega_{1}$, we make use of the continuous prolongation of the global solution from $\Omega_{11}$ to $\Omega_{12}$ and we apply the additivity of the integral with respect to domain $\Omega_{1}=\Omega_{11} \cup \Omega_{12} \cup \Gamma$. Denoting by $\left\{U_{1}\right\}$ the degrees of freedom vector associated to domain $\Omega_{1}$, this gives us:

$$
\left[K_{1}\right]\left\{U_{1}\right\}=\left[\bar{K}_{11}\right]\left\{U_{1}\right\}+\left[\bar{K}_{12}\right]\left\{U_{1}\right\} .
$$

In the above equation, $\left[\bar{K}_{11}\right]$ and $\left[\bar{K}_{12}\right]$ are the extensions to $\Omega_{1}$ of the classical stiffness matrices $\left[K_{11}\right]$ and $\left[K_{12}\right]$ related to subdomains $\Omega_{11}$ and $\Omega_{2}$, respectively. They formally contain the classical stiffness matrices and are completed with zero values to make them 
the same dimension of $\left\{U_{1}\right\}$. As well, we define $\left\{F_{1}\right\}=\left\{\bar{F}_{11}\right\}+\left\{\bar{F}_{12}\right\}$ the load vector associated to domain $\Omega_{1}$. The equality (7) is used to expand the Neumann problem from $\Omega_{11}$ to $\Omega_{1}$. It results in the following fixed point algorithm that can be implemented for the resolution of the coupling problem: for the $n$th iteration, starting with initial guesses $\{\Lambda\}^{(0)}$ and $\left\{U_{1}\right\}^{(0)}$, we look for $\left\{U_{1}\right\}^{(n)},\left\{U_{2}\right\}^{(n)}$ and $\{\Lambda\}^{(n)}$ such that:

1. Resolution of a Neumann problem over $\Omega_{1}$ :

$$
\left[K_{1}\right]\left\{U_{1}\right\}^{(n)}=\left\{F_{1}\right\}-\left[C_{1}\right]^{T}\{\Lambda\}^{(n-1)}+\left(\left[\bar{K}_{12}\right]\left\{U_{1}\right\}^{(n-1)}-\left\{\bar{F}_{12}\right\}\right)
$$

2. Resolution of a Dirichlet problem over $\Omega_{2}$ :

$$
\left[\begin{array}{cc}
{\left[K_{2}\right]} & -\left[C_{2}\right]^{T} \\
-\left[C_{2}\right] & {[0]}
\end{array}\right]\left\{\begin{array}{c}
\left\{U_{2}\right\}^{(n)} \\
\{\Lambda\}^{(n)}
\end{array}\right\}=\left\{\begin{array}{c}
\left\{F_{2}\right\} \\
-\left[C_{1}\right]\left\{U_{1}\right\}^{(n)}
\end{array}\right\} .
$$

We note that the mortar operator $\left[C_{1}\right]$ simply consists of the prolongation of former operator $\left[C_{11}\right]$ from $\Omega_{11}$ to $\Omega_{1}$. From a mechanical point of view, $\{\Lambda\}$ and $\left(\left[\bar{K}_{12}\right]\left\{U_{1}\right\}-\left\{\bar{F}_{12}\right\}\right)$ represent interface reaction forces (at $\left.\Gamma\right)$. More precisely, $\{\Lambda\}$ corresponds to the force of the local model whereas $\left(\left[\bar{K}_{12}\right]\left\{U_{1}\right\}-\left\{\bar{F}_{12}\right\}\right)$ is produced by the covered part of the global model (part $\Omega_{12}$ ). Figure 2 illustrates the procedure. Global and local problems are solved alternatively and only displacement and force exchanges at interface $\Gamma$ are required. The convergence test usually used to stop this algorithm relies on the equilibrium of the reaction forces at the interface of the two models.

Algorithm (8)-(9) constitutes what is now referenced as the non-intrusive coupling strategy in the literature. Since the global model is unmodified, the global stiffness operator can be assembled and factorized only once during the pre-processing step. More

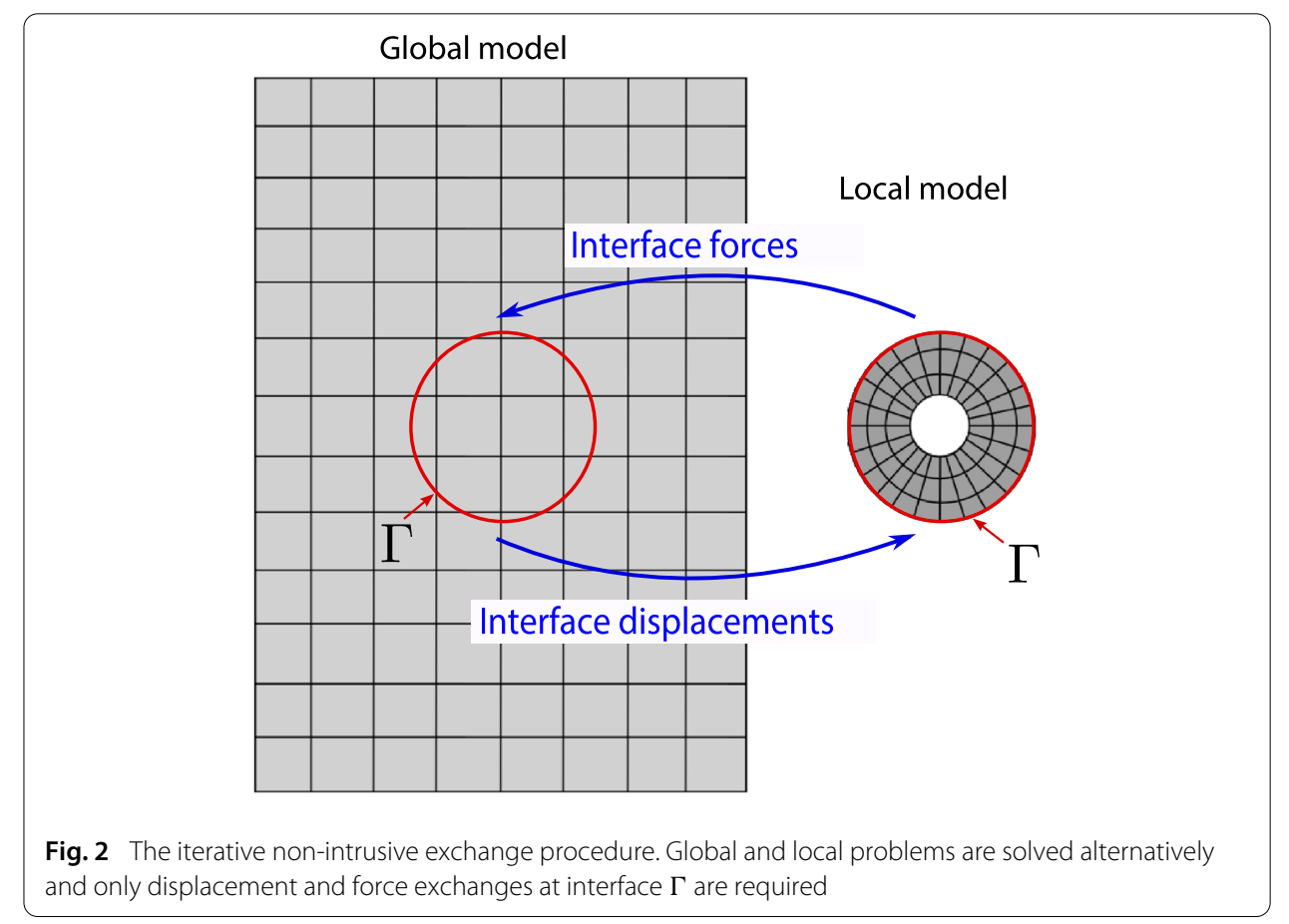


than a technique to couple different models, the method also offers the possibility to simply couple different numerical codes. Indeed, the global and local problem being solved alternatively, an industrial code can be used for the large-scale computation while a dedicated research code can be set up to solve a specific local behavior. The price to pay to reach non-intrusiveness is the number of iterations but this one can be deeply reduced by means of accelerations techniques, such as based on an Aitken's Delta Squared method or a Quasi-Newton method (see, e.g., $[10,20,34]$ ).

Remark 1 It may be noticed that the first iteration of the non-intrusive algorithm exactly corresponds to the so-called submodeling approach (see, e.g., $[7,8]$ ). Unlike the considered non-intrusive method, these submodeling approaches typically used in industry may generate important errors because of the lack of correction of the global model (see, e.g., $[9,10])$.

\section{Implementation issues}

Depending on the discretization of the two subdomains $\Omega_{11}$ and $\Omega_{2}$ along interface $\Gamma$, four distinct coupling situations may be listed (see Fig. 3 for illustration). We follow the nomenclature introduced in [33]. From the most restrictive to the most general case, we may encounter the situations of:

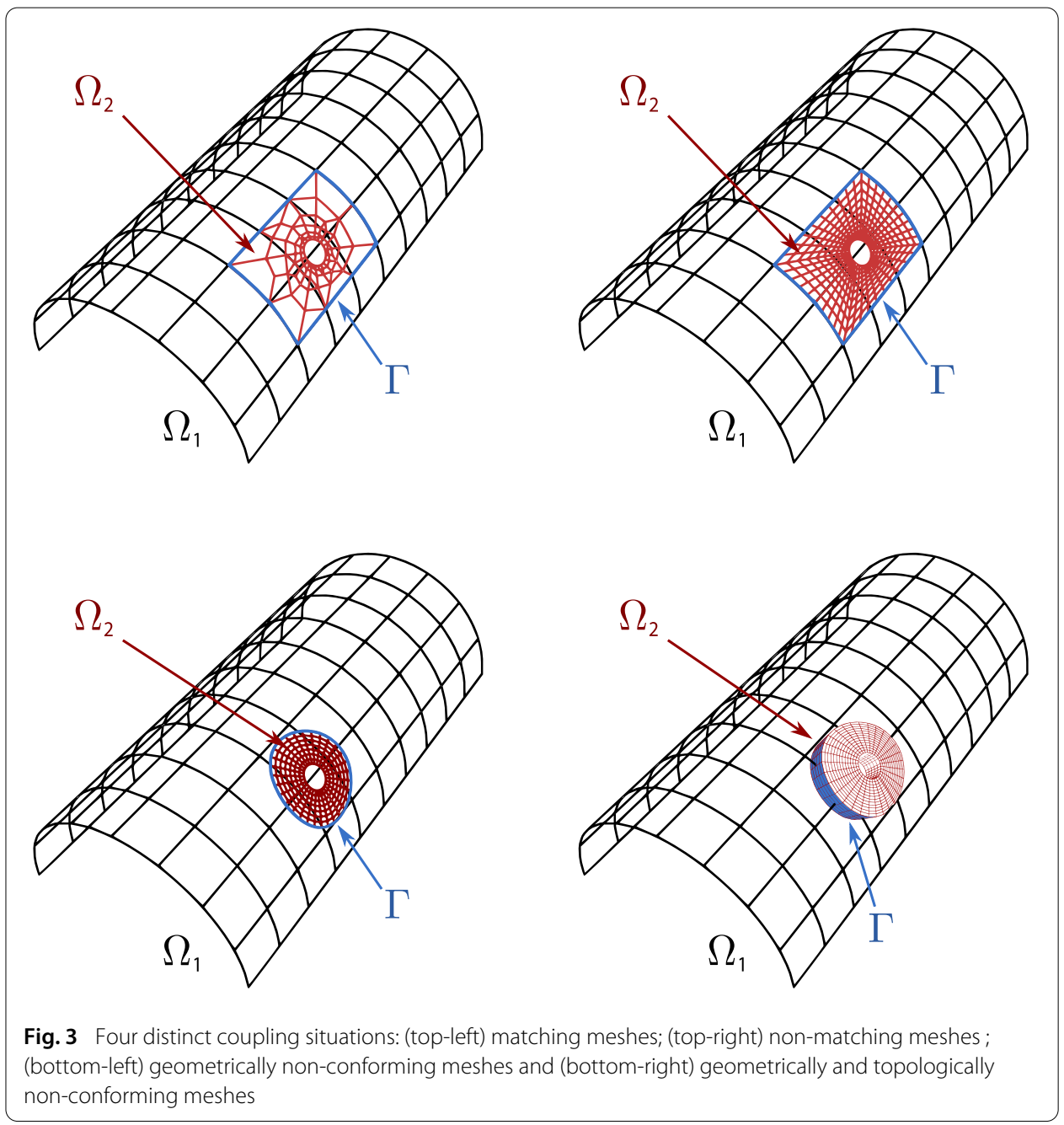


1. Matching meshes: the interface $\Gamma$ is aligned with the edges of the elements and the nodes of both models are coincident at the interface (see Fig. 3 (top-left)).

2. Non-matching meshes: the interface $\Gamma$ is aligned with the edges of the elements but the interface nodes of both models are shifted (see Fig. 3 (top-right)).

3. Geometrically non-conforming meshes: the interface $\Gamma$ is not aligned with the edges of the coarse elements which means that some elements of the global model are overlapped (see Fig. 3 (bottom-left)).

4. Geometrically and topologically non-conforming meshes: the interface $\Gamma$ is not aligned with the edges of the coarse elements, and the global and local models do not have the same topology along $\Gamma$ (e.g., shell on the global side and solid on the local side, see Fig. 3 (bottom-right)).

In the first contributions on non-intrusive coupling [10], the simplifying assumption of meshes and nodes compatibility was present. Then, interface projections were performed, which enabled to successfully handle non-matching meshes [16,20,34]. For simplicity, it was recommended to consider the trace along the coupling interface $\Gamma$ of the basis functions of the local model for the construction of $\mathcal{M}^{h}$. Until now, it seems that the implementation is mostly made in these two coupling situations, which evidently limits the practicability of the method as it is desired to achieve a satisfactory level of flexibility in defining the region of interest for a more detailed analysis. The difficulty when facing geometrically non-conforming meshes relies on the evaluation of the mortar operators $\left[C_{1}\right]$ and $\left[C_{2}\right]$ (see Eq. 6 ) and the stiffness matrix $\left[\bar{K}_{12}\right]$ (see Eq. 8), which would require to set up specific quadrature rules to integrate over pieces of coarse elements. A methodology based on the transfer of the local quadrature rule in the global computations has been proposed in [32,33] for the coupling of NURBS isogeometric models. Nevertheless, this approach appears inconsistent with the use of standard industrial FE codes. In addition, it has to be recalled that in the applications intended in this work, a global/local topologically non-conforming interface is to be investigated. This raises additional implementation issues. A framework for the proper non-intrusive coupling of a global plate model with local solid elements has been proposed in $[18,19]$, but this one relies on a complex procedure involving the definition of a cumbersome buffer zone. On top of that, it has to be underlined that, according to our knowledge, the simulation of a complex fully non-planar shell/solid coupling model by means of a non-intrusive procedure have not been carried out yet.

\section{The proposed methodology}

In this work, a pragmatic approach is employed as a solution of the aforementioned implementation issues. For the best possible satisfaction of non-intrusiveness, the strategy makes extensively use of existing tools in industrial FE codes. The procedure is depicted in Fig. 4. The idea is very simple: starting with a free independent local solid model (see Fig. 4a), it is proposed to modify its geometry in order to recover the robust situation of a geometrically and topologically conforming global/local interface. This goal is achieved by generating a surface transition mesh (i.e., made of shell elements). First, a grid intersection problem [35] is solved as classically in such methods in order to determine which are the elements from the global model that intersect the local model (see Fig. 4b). The region obtained by collecting these coarse elements forms the patch. The patch defines a new 


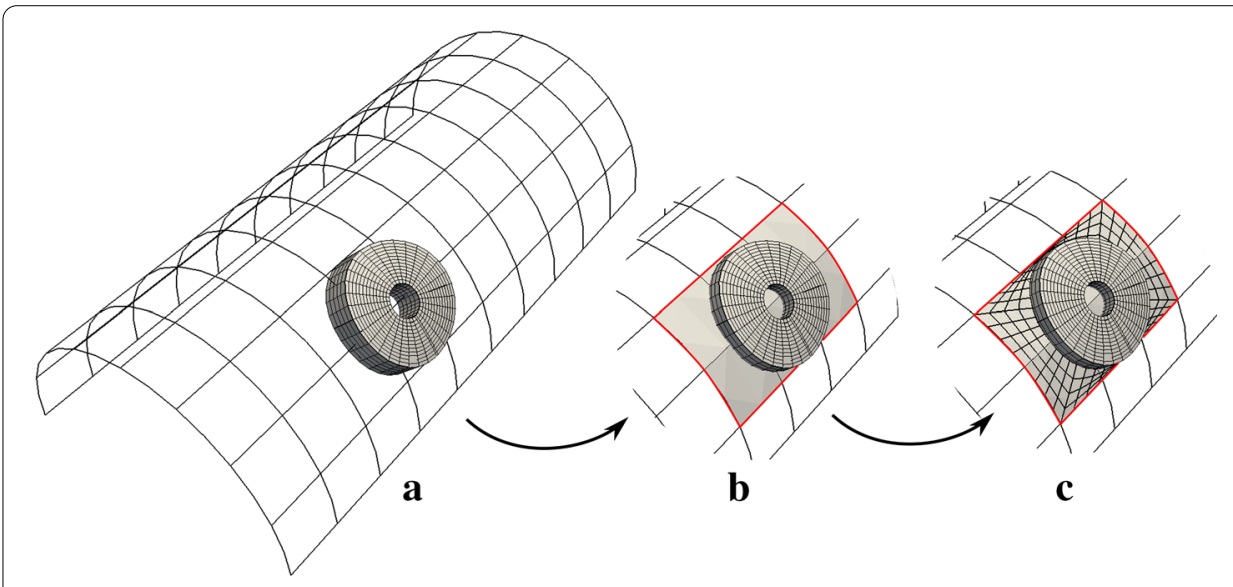

Fig. 4 Illustration of the proposed pragmatic strategy based on a transition mesh generation. Starting from a the initial meshes, step $\mathbf{b}$ : determination of the patch (grey area) and a new interface (red line) and step c transition mesh generation making appear two distinct interfaces (red line: global/local, blue line: shell/solid)

interface that corresponds to a set of edges from the global model, which, in turn, means that it is geometrically and topologically conforming with the global model. The second step of the approach is thus dedicated to the expansion of the local model to the new interface by means of a surface transition mesh. As a consequence, it becomes possible to perform the global/local exchanges as classically across the new geometrically and topologically conforming interface (see Fig. 4c).

More precisely, the proposed strategy makes appear two distinct interfaces which enables to separate the difficulties due to the combination of the global/local and shell/solid characters of the coupling. The shell/solid coupling is managed within the patch (see blue line in Fig. 3 (bottom-left)) while the global/local exchange is performed by means of a shell/shell coupling at the patch boundary (see red line in Fig. 4c). This offers the possibility to use standard methods employed in industry to connect shell and solid elements, thus avoiding to introduce cumbersome buffer zones.

\section{Implementation}

Unlike the previous section, which presents the proposed strategy in a general way, this section specifies the particular use of the Abaqus software suite for the finite element analysis. The chosen software is one of the most employed in the aerospace industry, being used in the operational environment of Airbus Group.

\section{Co-simulation derived implementation path}

The co-simulation technique is an analysis feature proposed by SIMULIA that allows coupling different $\mathrm{FE}$ analysis and managing data exchanges between user-defined domains. Quantities to be exchanged and time-synchronization during analysis can be defined by the user in a specific co-simulation configuration file. Abaqus v6.14 co-simulation native capabilities have been extended through additional specific developments (user subroutine and Abaqus python script for time synchronization of global/local jobs). This environment was used for replicating a non-intrusive algorithm, while accounting for meshing strategy exposed hereabove. 
The proposed meshing strategy is applied through a main python script for Abaqus GUI pre-processor (CAE), that handles global and local model preparation (see Fig. 5). Then the computation is managed by the Abaqus co-simulation engine and additional features mentioned hereabove, implementing the non-intrusive coupling strategy, defined by algorithm (8)-(9). As stated earlier, the convergence of the iterative algorithm is tested regarding the equilibrium of reaction forces at the interface of the models, also known as residual efforts. The main script also contains in its beginning the instantiation operation of the implemented classes, namely the coupling classes, the global model class and the local model class. Those operations in the beginning of the script correspond to the resolution of the grid intersection problem, the transition mesh generation, the global and local model data initialization and the determination of the connectivity between both models. "Transition mesh generation" section is dedicated to explain with more details the operations related to the transition mesh generation.

After the initial operations, the non-intrusive coupling algorithm itself begins. The exchange procedure exactly follows the one described in Fig. 2. The first step is the resolution of the global problem (8). In order to do so, a new input file for the global model is created so that the loading corresponding to the interface forces is considered, as this problem has Neumann conditions on $\Gamma$. Then, the script calls upon a series of Abaqus functionalities in order to create and submit a job containing the required analysis, as well as to access the postprocessing tools required to find the interface displacements so that they can be imposed as boundary conditions in the Dirichlet problem that follows. After solving the global problem and before entering the local one, a projection is necessary in order to translate the interface displacements from the global model into the local one by using the information of connectivity between both models previously assembled.

The next step is the resolution of the local problem (9). Once more, a new input file is created, but now the local model with the transition mesh added is considered, so that the
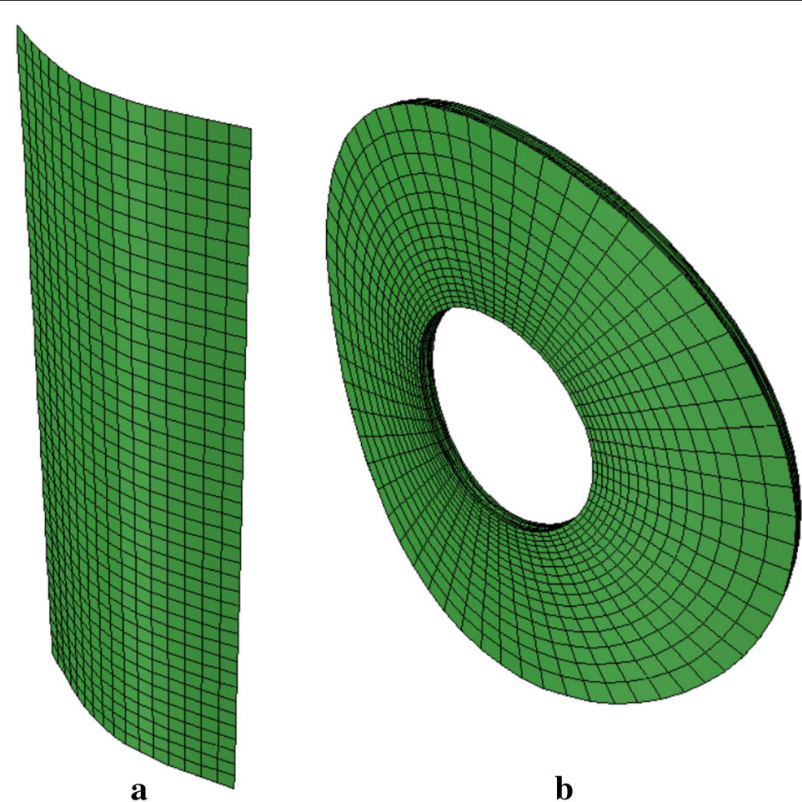

b

Fig. 5 Cylindrical global and local initial models: a global model with shell elements and $\mathbf{b}$ local model with hexahedral elements 
interface displacements are taken into account as boundary conditions, as this problem is a Dirichlet one. Again, the script calls upon a series of Abaqus functionalities in order to create and submit a job with the required analysis, as well as to find the reaction forces at the interface so that they can be imposed as loading in the Neumann problem in the next iteration. After solving the local problem, another projection is necessary in an inverse way, so that the efforts are translated from the local model into the global one.

The reaction forces at the interface that were just determined are used to calculate the residual forces, which correspond to the unbalance between the reaction forces in both models. These residual forces are taken as the convergence parameter of the method.

Following the overview of the implementation of the proposed strategy, the next section proceeds to explain in a more detailed way the fundamental aspect of this work, namely the non-planar transition mesh generation. This operation is made only once at the beginning of the algorithm as we do not consider expansion of the local model in this work (e.g., due to crack or damage propagation). Nevertheless, given the ease of use of the procedure, we insist that it could be repeated at each iteration if necessary.

\section{Transition mesh generation}

The transition mesh is generated through the Abaqus scripting interface so that the procedure is automated. A Python script includes several commands that call a series of Abaqus functionalities. For illustration purpose, we consider a structure with a cylindrical geometry (see Fig. 5).

Once the patch is determined (see Fig. 6), the operations for the generation of the transition mesh are applied. The method begins by accessing the model database corresponding to the local model and creating an object that contains all the information relative to the model. Knowing that a model in Abaqus is constituted by individual parts, the initial local model information was present in a single part. Then, a new part is created in order to generate the transition mesh, which is assembled in a later operation. In this new part, an offset plane is defined according to the cylinder radius, corresponding to the projection of the cylinder into a plane. Only the patch needs to be projected.

A sketch is created using the plane just defined and the edges corresponding to the inner and the outer boundaries of the transition region are generated. The inner boundary is characterized by the initial local model considering its mid-plane while the outer boundary is defined by the interface corresponding to the global model patch border. All of those boundary edges were previously determined by the script and, in the case of the cylinder, they were projected into the plane in order to allow a proper mesh generation (see Fig. 7), that is subsequently reprojected into the cylindrical geometry.

After the generation of the shell geometry by the planar sketch (see Fig. 7a), each edge is seeded with a fixed number of elements (see Fig. 7b). When the ratio between global and local elements becomes too large, meshing constraints may appear. This may lead to either a distorted or oversized transition mesh. In order to avoid these constraints, we use non-matching meshes at the new coupling interface. This can be performed very conveniently using Mortar operators, that are explicit here, since the meshes are nested.

Then, the mesh is generated in that new part (see Fig. 7c) and both parts are assembled. An input file is written by an Abaqus job, then the Python script reads it in order to perform the projection of nodes and elements to the cylindrical geometry, manually 


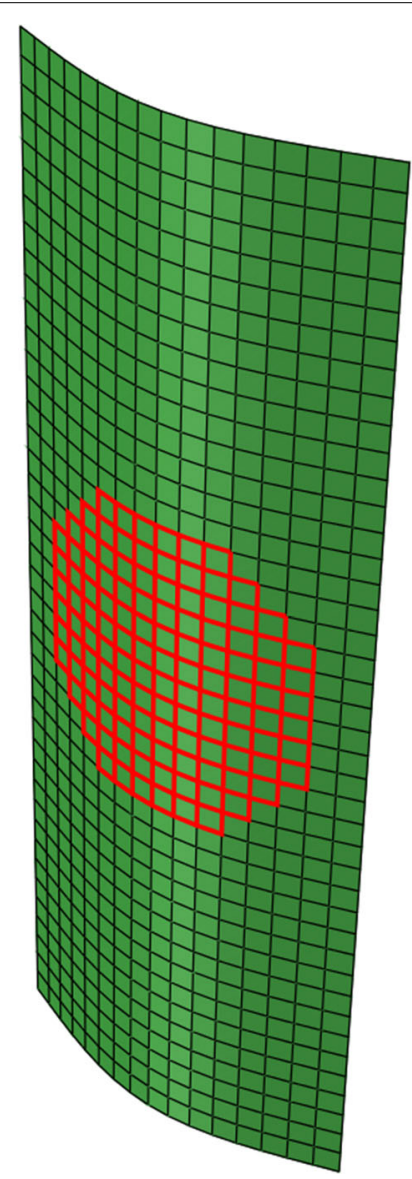

Fig. 6 Selection of the patch. A integer number of global elements are selected as they will be substituted by the refined local patch

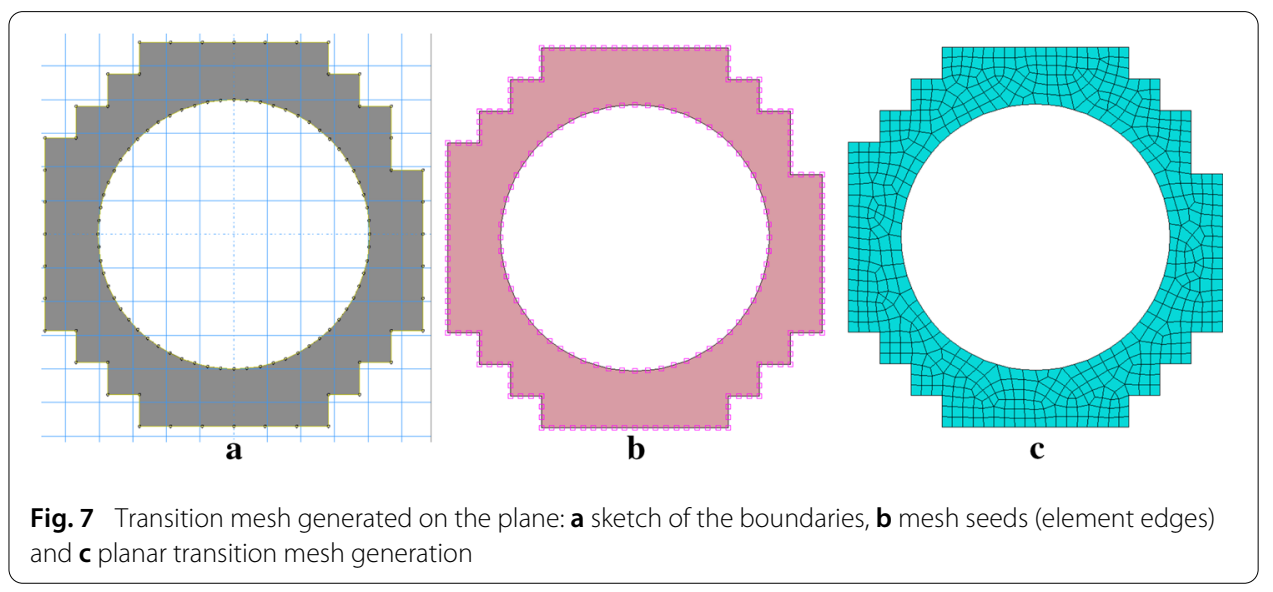

writing a new input file after that projection. The next step involves the creation of a new model from the last input file that was written. The material properties are copied from the old model, while the parts of the new model are assembled. Figure 8 illustrates the model. 


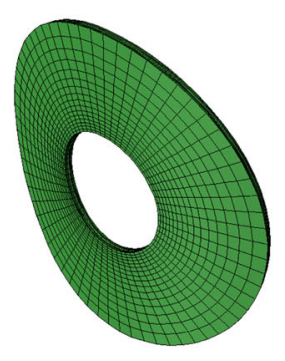

a

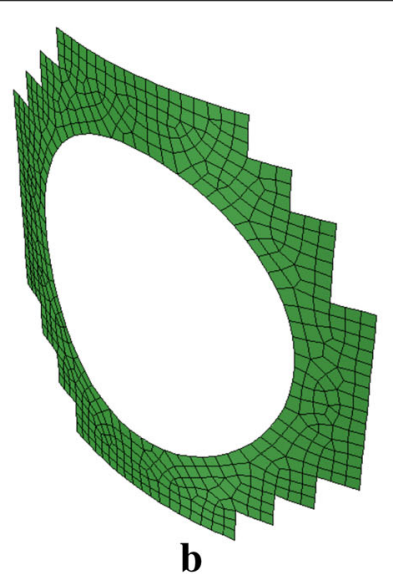

b

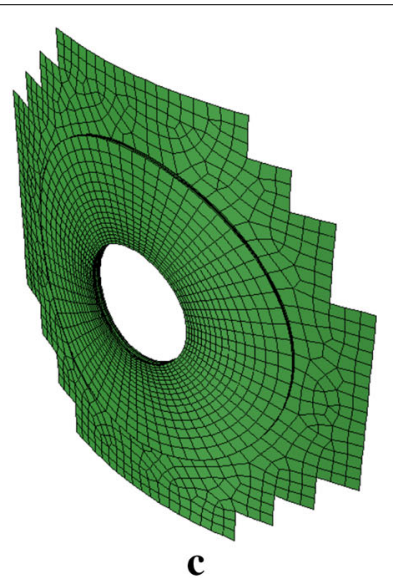

C

Fig. 8 Cylindrical local model modification: a initial local model, b non planar transition mesh and c transition mesh included in the local model

The last procedure is the implementation of the Abaqus based shell-to-solid coupling (see Abaqus documentation [36]: "Shell-to-solid coupling" section), what requires the knowledge of the shell edges corresponding to the inner boundary of the transition mesh and the outer elements of the local solid model. Thus, a new input file is generated considering the shell/solid coupling.

\section{A first validation example}

As a first demonstration and validation, we now carry out two numerical experiments based on an academic example. The layered cylindrical shell panel weakened by a small circular hole, as previously introduced in "Transition mesh generation" section is considered. The interest of this test case is twofold: on the one hand, it collects all the implementation difficulties: non-conforming geometries, shell-to-solid coupling, fully non-planar structure, etc; and on the other hand, numerical and modeling references are available. To start with, the composite shell is studied in the elastic regime which enables to evaluate the quality of the Abaqus built in shell-to-solid coupling. Then, a dedicated model for the non-linear behavior of composite materials is implemented in order to compute the local damage of the structure.

\section{Cylindrical composite shell: elastic case}

The coordinate system, geometry, stacking sequence, and boundary conditions regarding the investigated test case are given in Fig. 9. It concerns a composite material made of carbon fibers with a $\left[45^{\circ}, 90^{\circ},-45^{\circ}, 0^{\circ}\right]_{\text {sym }}$ stacking sequence (total thickness $1.0 \mathrm{~mm}$ ); $0^{\circ}$ refers to $\mathrm{X}$ axis (vertical ascending), and $\mathrm{Z}$ is the normal direction to the surface cylinder. The bottom end of the cylinder is clamped while a vertical $0.45 \mathrm{~mm}$ translation is imposed on its top end. For the simulation, the meshes of Fig. 5 are considered for the global shell and the local solid models. At this time, we assume no damage in the composite: in the local model, only the plies (without interfaces) are modeled with a linear orthotropic elastic behavior (see Table 1 for the values of the material properties). In Abaqus, each ply is modeled with a layer of C3D8 elements, which is the typically used 8-node brick element for the investigation of the behaviors of meso-constituents within a laminate. For 


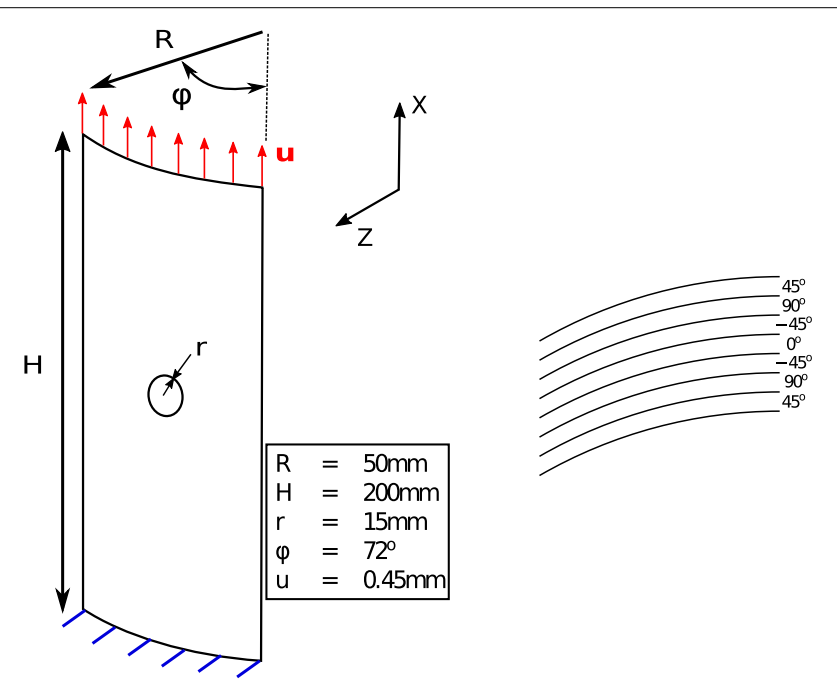

Fig. 9 Description and data of the cylindrical test case. Geometry and loading (left) and stacking sequence (right)

Table 1 Elastic properties of the plies

\begin{tabular}{|c|r|}
\hline \hline$E_{1}(\mathrm{GPa})$ & 154.00 \\
\hline$E_{2}(\mathrm{GPa})$ & 9.00 \\
\hline$E_{3}(\mathrm{GPa})$ & 9.00 \\
\hline
\end{tabular}$\quad$\begin{tabular}{|l|l|}
\hline$\nu_{12}$ & 0.32 \\
\hline$\nu_{13}$ & 0.32 \\
\hline$\nu_{23}$ & 0.40 \\
\hline
\end{tabular}$\quad$\begin{tabular}{|c|c|}
\hline$G_{12}(\mathrm{GPa})$ & 5.00 \\
\hline$G_{13}(\mathrm{GPa})$ & 5.00 \\
\hline$G_{23}(\mathrm{GPa})$ & 3.30 \\
\hline
\end{tabular}

the shell, an homogenized behavior is adopted; given the stacking sequence, this one is quasi in-plane isotropic.

As a preliminary study, we compare the computed global-shell/local-solid non-intrusive solution with a reference one obtained with a full 3D model made only of solid elements (see Fig. 10a). The paths represented in red in Fig. 10 gather the nodes where the stress tensor components will be compared for both models. It has to be noticed that each ply has two correspondent paths, one in the upper and one in the lower limit along the thickness. Figure 11 contains the stress components distribution along the lower path of a ply at $90^{\circ}$. Those results present essentially the same behavior as the ones in other plies and for other path directions. For the shell/3D model, the stresses are taken only in the solid elements, which means that the left extremity corresponds to the hole and that the right one is associated to the shell/solid interface.

Except at the right extremity, the two curves are very close which accounts for the accuracy of the proposed strategy. Near the shell/solid interface, a discrepancy can be observed, being even more relevant for the out-of-plane stresses. This phenomenon is not physical and is inherent to the different topological models used for the global/local simulation. Indeed, since the shell model constitutes a surface approximation of the 3D solid model, it is expected not to exactly reproduce the solid behavior close to the shell/solid coupling. As a remedy, a sophisticated 3D recovery technique has been proposed in [18] but still, a buffer zone was required to alleviate these edge effects. In our intended application, if we consider that the composite material may be subjected to damage in the region of the interface stress concentration, we introduce an erroneous source of degradation that has the potential to completely change the comportment of the structure. The pragmatic chosen strategy to that problem is the adoption of an elastic layer that spans around the 


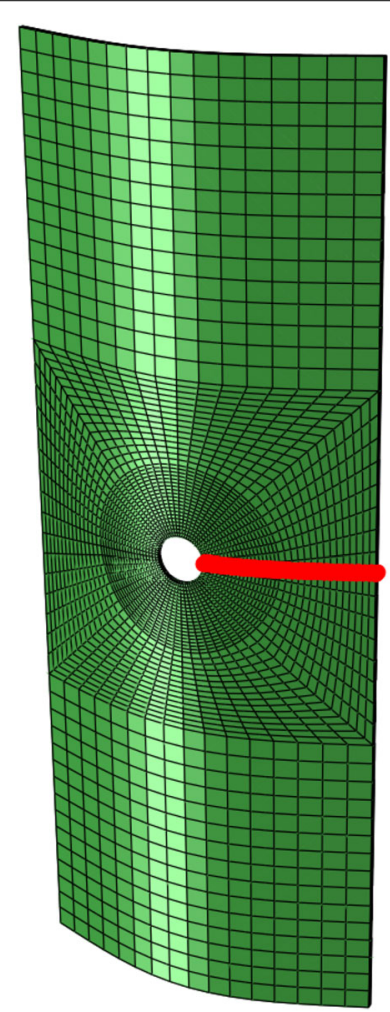

$\mathbf{a}$

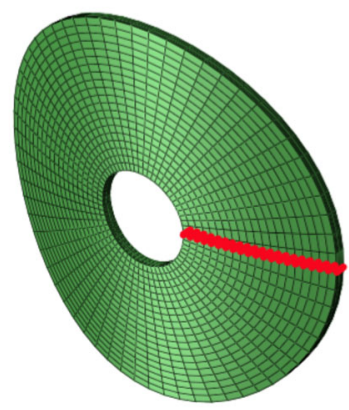

b

Fig. 10 Stress analysis path. Paths where the stress components are compared for the full 3D reference model (a) and the 3D part of the global/local model (b)

interface. As a consequence, the material may have non-linear properties of damage only in the inner portion of the solid region, preventing the generation of degradation caused by the shell/solid coupling. The elastic layer solution appears consistent (especially in our case of an elastic global model), and we emphasize that it constitutes a common industrial practice.

\section{Cylindrical composite shell: damage}

We now reanalyze the cylindrical test case by considering the local damage of the composite around the hole. This test case was selected for its specific sensitivity to local nonlinearities: in particular, the displacements in the normal direction to the surface cylinder severely depend on damage in central area. The geometrical parameters, prescribed displacements and elastic properties are the same as for the previous numerical experiment. Abaqus COH3D8 elements (i.e., 8-node three-dimensional cohesive elements) are added to model the interfaces between the plies. A dedicated model for the non-linear behavior of the composite material is derived from Progressive Failure Analysis (PFA) through a dedicated ABAQUS user-subroutine (see [37]). Table 2 presents the numerical values considered for the damage model. Since our interest in this work is on the development of an efficient coupling strategy but not on the advanced modeling of damage, the model considered is a rather simple one. The elastic moduli within plies are affected by knockdown factors that depend on stress state within the material, evolving between 0 (no 

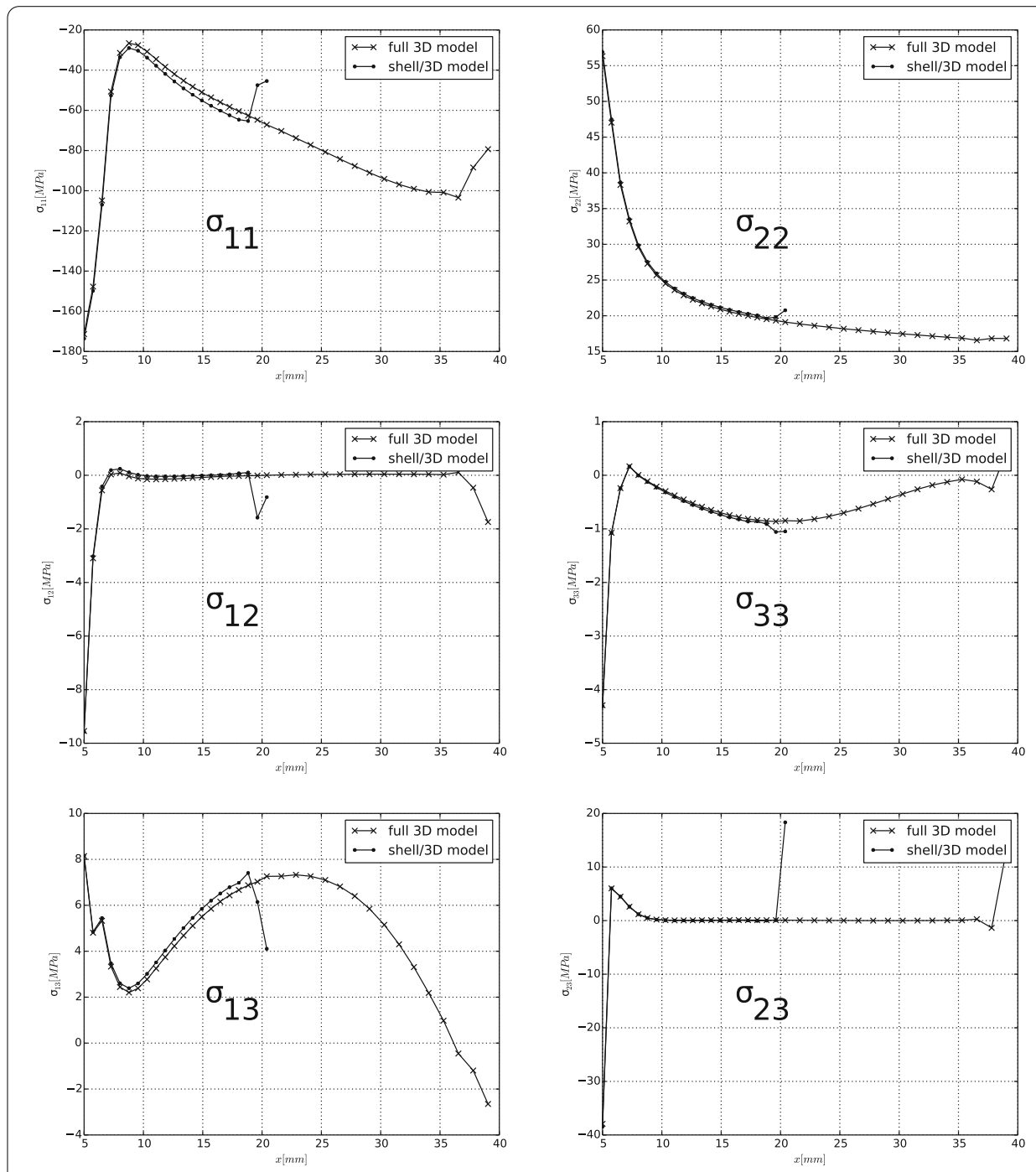

Fig. 11 Stress components. Evolution of the six components of stress along the above paths (ply at $90^{\circ}$ )

damage) and 1 (when reaching failure stress). The elastic moduli within interfaces depend on two quantities: interfacial stress states, relatively to interfacial strengths (analogous PFA framework as for plies); and damage state reached within neighboring plies (which allows to capture coupling effects between transverse cracks and delamination). With such parameters and the application of the vertical $0.45 \mathrm{~mm}$ translation, we make sure that the stress threshold for damage is exceeded.

For a proper comparison, the test case is computed through four modeling paths (see Fig. 12):

1. The top-down submodeling approach, Fig. 12a, serving as the industrial state-of-theart method. Indeed, it currently constitutes the common practice in industry. From algorithm (8)-(9), it corresponds to perform the first iteration only, without going back to the global model (see, e.g., $[7,8]$ ).

2. The proposed iterative non-intrusive approach, Fig. $12 \mathrm{~b}$, conducted accordingly with algorithm (8)-(9) (6 iterations were needed to reach convergence). 
Table 2 Material properties: ply failure stresses $\sigma_{i j+}^{l i m}$ and $\sigma_{i j-}^{l i m}(\mathrm{MPa})$, interface elastic moduli $k_{i j}(\mathrm{~N} / \mathrm{mm})$ and interface failure stresses $\sigma_{i j}^{l i m}(\mathrm{MPa})$

\begin{tabular}{|l|r|}
\hline \hline$\sigma_{11+}^{l i m}$ & +2500.0 \\
\hline$\sigma_{11-}^{l i m}$ & -1500.0 \\
\hline$\sigma_{22+}^{l i m}$ & +50.0 \\
\hline$\sigma_{22-}^{l i m}$ & -300.0 \\
\hline$\sigma_{33+}^{l i m}$ & +50.0 \\
\hline$\sigma_{33-}^{l i m}$ & -300.0 \\
\hline$\sigma_{12}^{l i m}$ & 100.0 \\
\hline$\sigma_{13}^{l i m}$ & 70.0 \\
\hline$\sigma_{23}^{l i m}$ & 70.0 \\
\hline
\end{tabular}$\quad$\begin{tabular}{|l|l|l|l|}
\hline$k_{33}$ & 100000.0 \\
\hline$k_{13}$ & 100000.0 \\
\hline$k_{23}$ & 100000.0 \\
\hline & \\
\hline & $\sigma_{13}^{l i m}$ & 60.0 \\
\hline
\end{tabular}

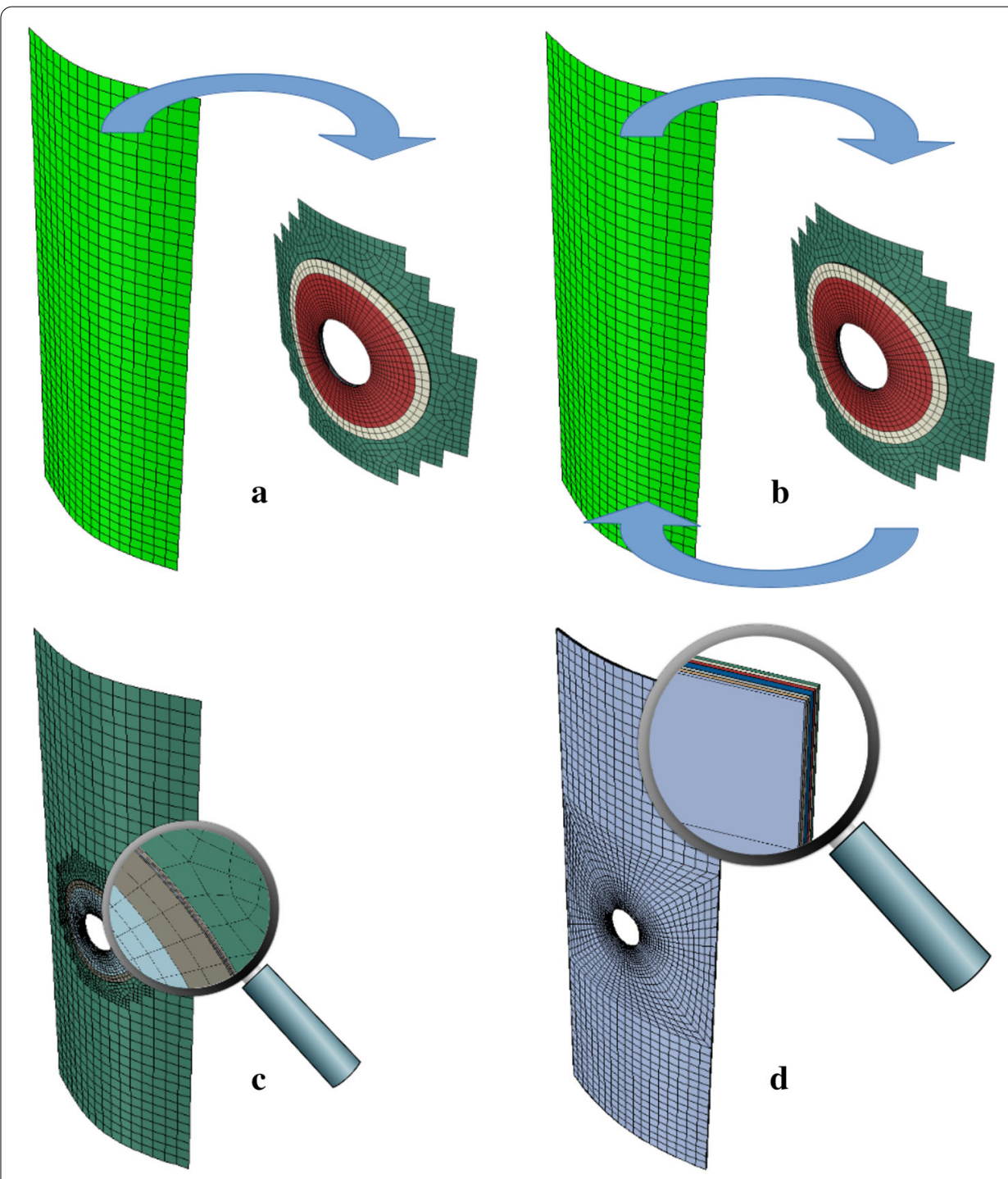

Fig. 12 Demonstration and validation test-case. Comparison of four approaches: a top-down submodeling, b iterative non-intrusive, $\mathbf{c}$ monolithic shell/solid and $\mathbf{d}$ full solid reference 
3. The monolithic shell/solid approach, Fig. 12c, serving as a numerical reference to the iterative solver of approach 2 . For this computation, the only difference compared to approach 2 is that system (5) is directly solved instead of performing algorithm (8)(9).

4. The monolithic full solid approach, Fig. 12d, serving as a modeling reference to approach 2. This model renews the one of Fig. 10a by taking into account damage throughout the whole structure.

For each model, the same time discretization in all non-linear computations is adopted (10 time increments). It has to be noted that when the simulation involves a local model, this one has been constructed using the proposed strategy based on the generation of a transition mesh. We clearly see that, thanks to this treatment, the global/local interface becomes conforming and only simple interface projections are needed to transfer the data between the two resulting, quasi-uniform, non-matching meshes. Furthermore, it has to be said that the transition mesh made of shell elements is considered elastic and that an additional layer of two elastic solid elements is set up (see grey region) in order to prevent from erroneous sources of degradation due to the Abaqus shell/solid coupling.

In terms of results, Fig. 13 shows the spatial distribution obtained for the displacement of interest, i.e. the displacement along the $\mathrm{Z}$ axis. At first glance, it can be observed that approaches 2, 3 and 4 allow recovering global effects from local non-linearities. However, this cannot be accounted for through the submodeling approach 1 that exhibits severe violation of the global equilibrium, due to the lack of correction of the global model. It has to be emphazised that not only the global solution but also the local solution appear erroneous due to this lack of force redistribution. Then, we can notice that approaches 2 and 3 exactly lead to the same solution. This was expected since it has already been proven that the non-intrusive iterative algorithm(8)-(9) converges to the monolithic solution of system (5) (see, e.g., [17]). Finally, it has to be noticed that the multiscale solution of approaches 2 and 3 looks very close to the one of approach 4 , which validate the global/local modeling set up to solve such a problem.

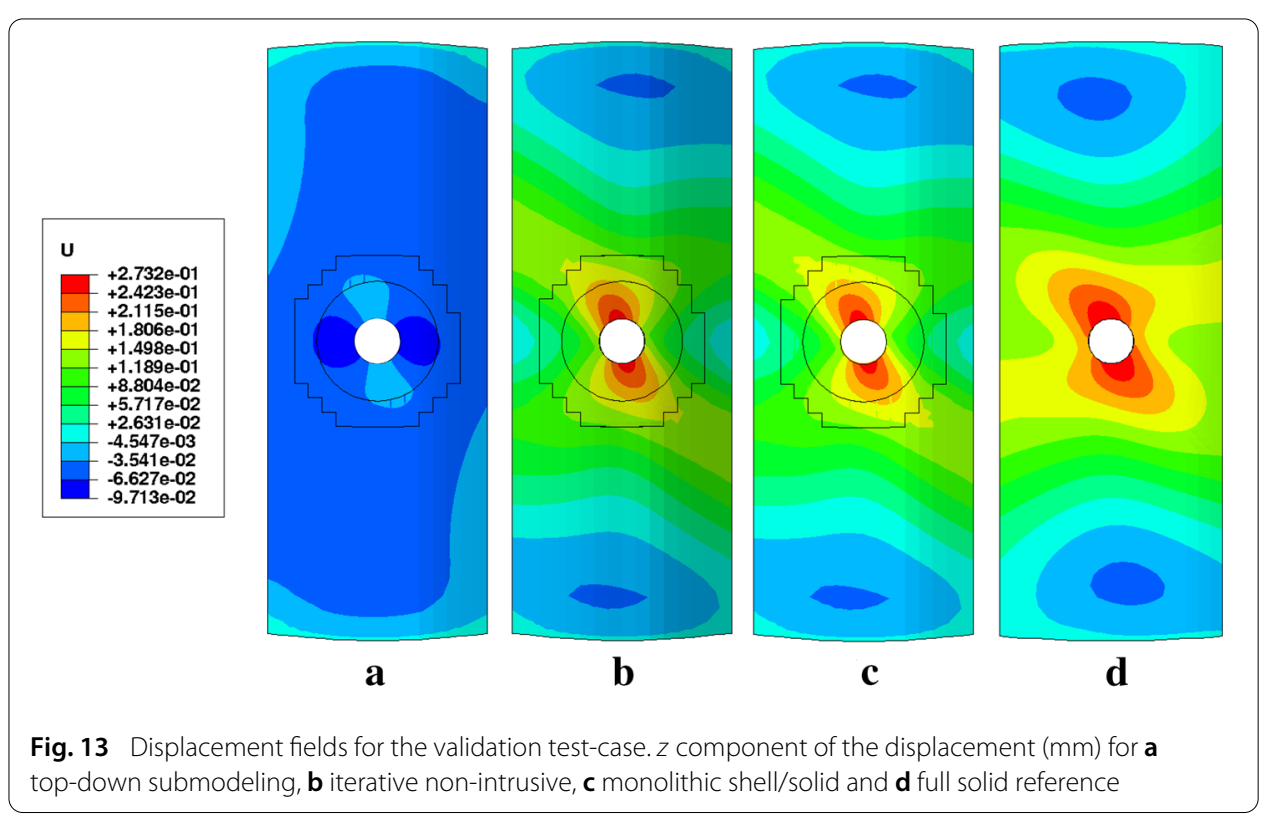


Remark 2 For approaches 1 and 2, one may notice that the global solution is available throughout the whole global shell; that is, also in domain $\Omega_{12}$ which is covered by the local domain. In the plots, we decided not to provide the global solution over $\Omega_{12}$ since it has no physical meaning (and it depends on the initialization).

To complete the study, Fig. 14 presents the distribution of the intra-laminar damage indicators in ply 4. For the monolithic full solid approach, a zoom around the hole is performed for a proper comparison with the other simulations. These results illustrate how the iterative process converges to local quantities of interest, which is the validation criterion for the approach. One may notice that no spurious damage can be observed at the border of the local zone, which accounts for the use of an additional elastic solid region to counterbalance the non-desirable effects of the Abaqus shell/solid coupling. Finally, the same behavior as for the displacements can be observed: the submodeling strategy is not able to properly compute the local damage of the structure while the iterative nonintrusive process 2 leads to the same solution as the reference monolitic coupling, and to a solution close to the brute force full 3D approach.

\section{Application to industrial realistic examples}

As an additional step towards the transfer of the method to the industrial environment, two representative use-cases extracted from engineering practices are now examined. The industrial background is as follows: during aircraft design and certification process, one of the most demanding demonstrations towards airworthiness authorities originates from structural survivability to engine burst. When accidental in-flight engine burst occurs, fuselage panels may be subjected to large cuts, thus severely affecting the skin and the stiffeners. As a result, evidence must be made that under such circumstances, remaining load-carrying capabilities are still enough to make the aircraft fly and land. For such demonstration, stress-offices are essentially relying on physical tests. Investigating the

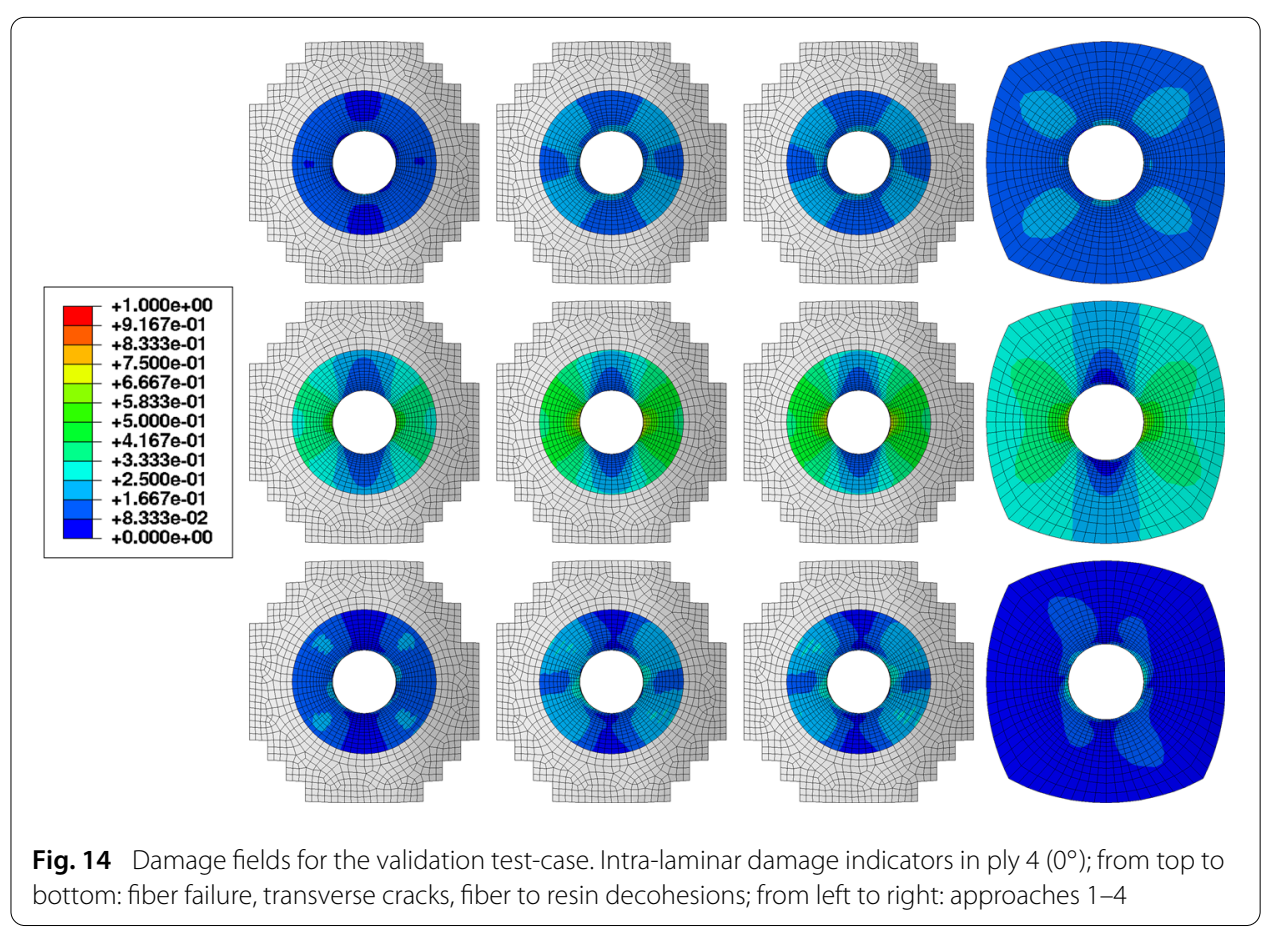


capabilities of the proposed method to provide a virtual assessment of the risks in such a context appears then of primary interest. It would offer the opportunity to alleviate part of expensive and time consuming physical tests from the design and certification process.

\section{Large cuts in stiffened panels}

For validation purpose, the stiffened panel test case depicted in Fig. 15 is first investigated. At this scale, a direct monolithic approach still remains affordable, which enables to properly study the validity and robustness of the method. A particular care has been taken to make the test case as representative as possible of actual aeronautical designs and engineering practices. The problem dimensions, boundary conditions and global/local modeling are summarized in Fig. 15. Connections of stiffeners to skin are modeled through kinematical constraints between nodes of stiffener flanges and skin. The panel is clamped on its bottom side and subjected to traction. A large horizontal cut affects the skin and the central stiffener. The same composite material as in "A first validation example" section is considered: it consists of a $\left[45^{\circ}, 90^{\circ},-45^{\circ}, 0^{\circ}\right]_{s y m}$ stacking sequence (ply thickness $0.125 \mathrm{~mm}$, $0^{\circ}$ refers to $\mathrm{X}$ axis, see coordinate system in Fig. 15). The structure is studied in its elastic regime, the objective being to make sure that the crack will not propagate due to too high stress states around the crack tips. The material properties are the same as in Table 1. A solid modeling is adopted around the crack tips while a homogenized elastic shell is considered everywhere else in the stiffened panel. Following the procedure proposed in this work, a transition shell mesh is generated on each side of the central stiffener to expand the two initial solid parts. The resulting global (shell)/local (shell) interface on which the non-intrusive coupling is performed is conforming and even matching in this situation.

The results of the corresponding non-intrusive global/local simulation are plotted in Figs. 16b, 17b and 18b in terms of global displacements, local displacements and local stresses, respectively. We clearly observe the strong coupling between the two scales in the situation of large cuts: the material state in crack tip areas severely influences the kinematics of the crack lips and thus the global structural behavior of the panel. Not only traction but also bending effects occur in the panel due to the middle crack. In addition, the industrially-used top-down submodeling approach and the reference monolithic strategy have been carried out for a proper assessment. The same conclusions as in "A first validation example" section can be drawn: the impact of the large cut on the global model is not accounted for with the submodeling approach while the iterative non-intrusive strategy accurately recovers the reference monolithic solution.

\section{Large cuts in a fuselage section}

For demonstration purpose, a fully representative fuselage section is finally investigated. The problem setup is given in Fig. 19. It concerns a real portion of an aircraft (about $7 \mathrm{~m}$ large) subjected to a large cut (length: $170 \mathrm{~mm}$, radius at crick tips: $1 \mathrm{~mm}$ ). Considered global FEM was extracted from actual sizing exercises practiced within Airbus stress offices. This modeling includes skin, floor, stiffeners and frames: its size $\left(>10^{6}\right.$ degrees of freedom) and complexity are thus fully representative of a situation where industrial time and cost constraints prohibit mesh refurbishments. As a result, only non-intrusive techniques can be implemented on such a model to perform a global/local simulation. 


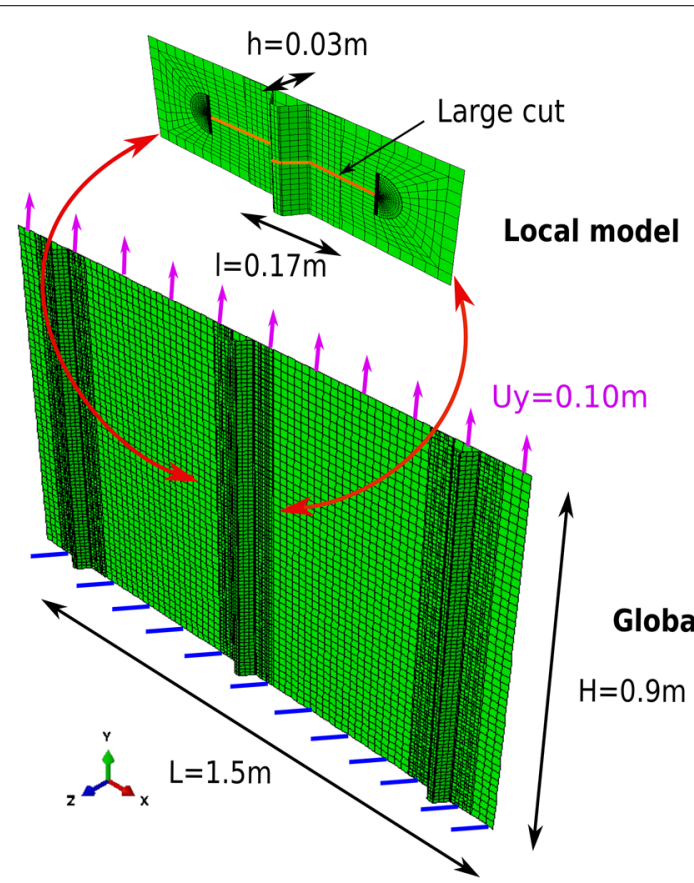

Fig. 15 Description and data of the stiffened panel test case. Geometry, loading and large cut definition
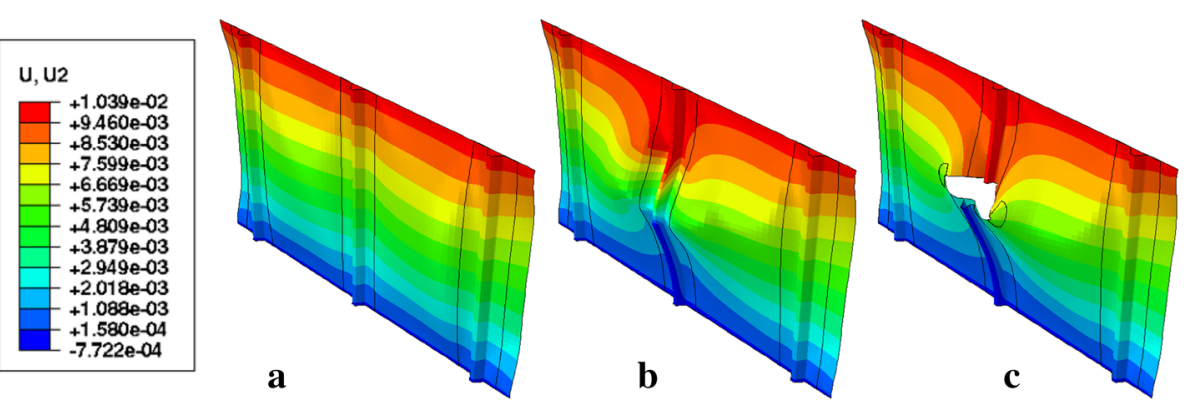

Fig. 16 Global displacement field for the stiffened panel subjected to a large cut. Vertical displacement (m) in the global model for a top down submodeling, $\mathbf{b}$ global model of the iterative non-intrusive, $\mathbf{c}$ monolithic reference
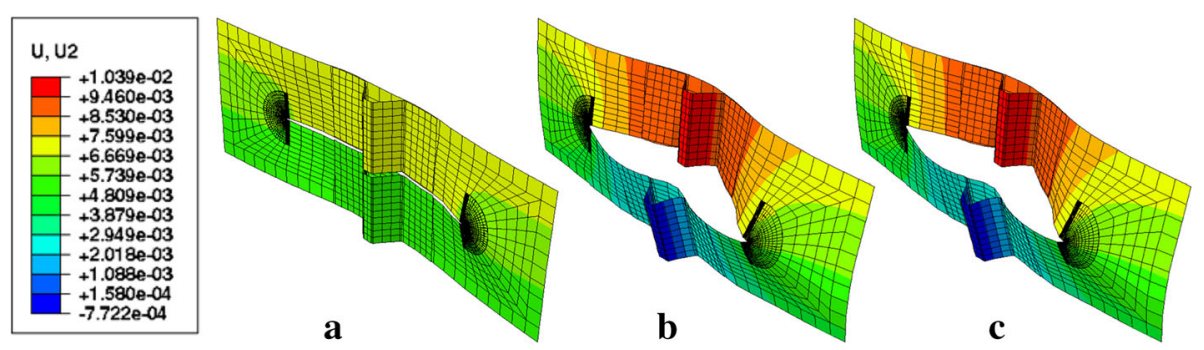

Fig. 17 Local displacement field for the stiffened panel subjected to a large cut. Vertical displacement $(m)$ in the local model for $\mathbf{a}$ top down submodeling, $\mathbf{b}$ local model of the iterative non-intrusive, $\mathbf{c}$ monolithic reference 

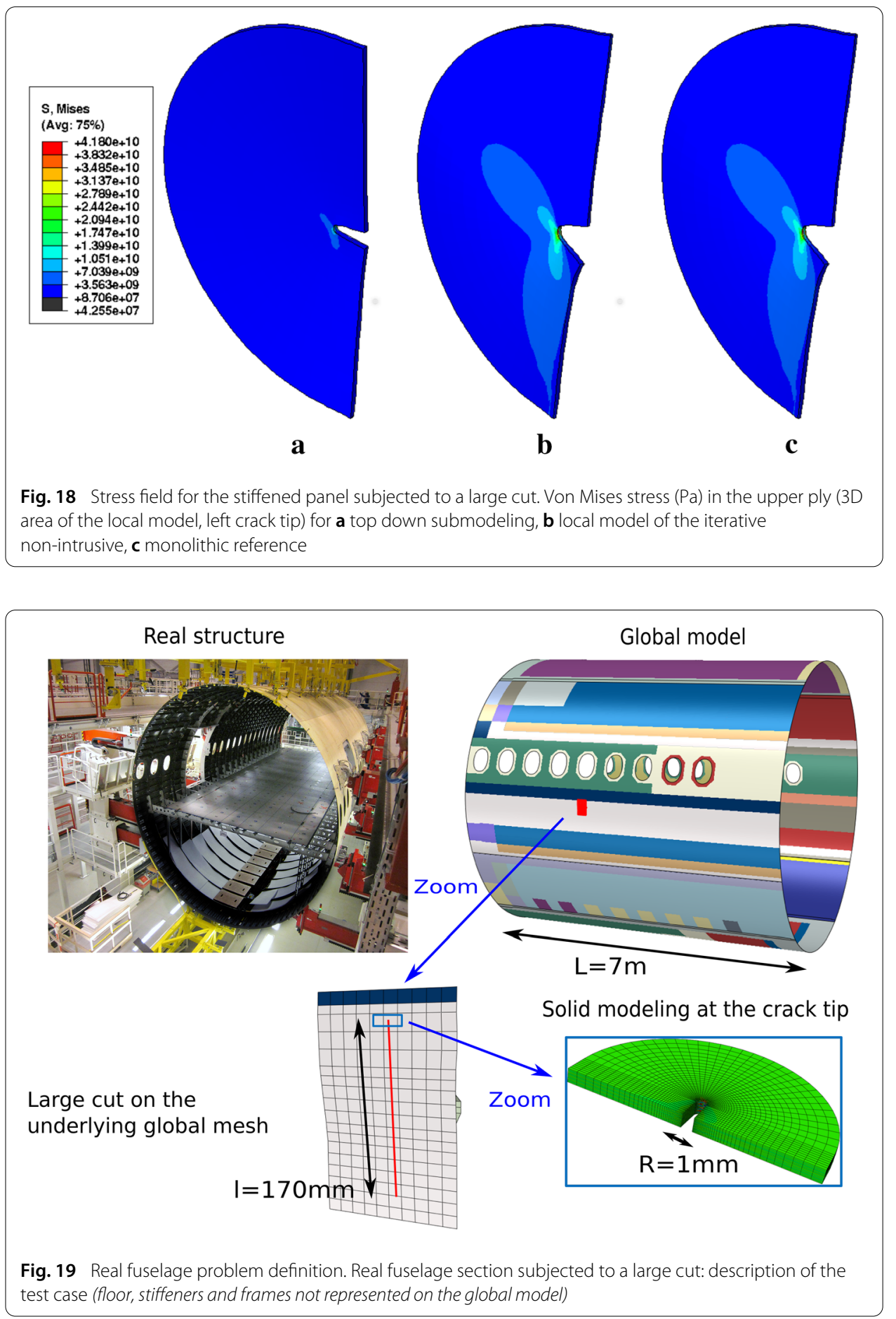

An analogous global/local modeling as for the stiffened panel of Fig. 15 is considered. The explored use-case is highly challenging for multiscale analysis: characteristic lengths at global and local scales are $7 \mathrm{~m}$ (fuselage section length) and $10 \mu \mathrm{m}$ (ply thickness) respectively. The fuselage is clamped on its left side and subjected to traction on the other side. An internal pressure is also prescribed so as to model the typical pressure gap encountered in flight. Figure 20 shows the results obtained with the proposed non- 

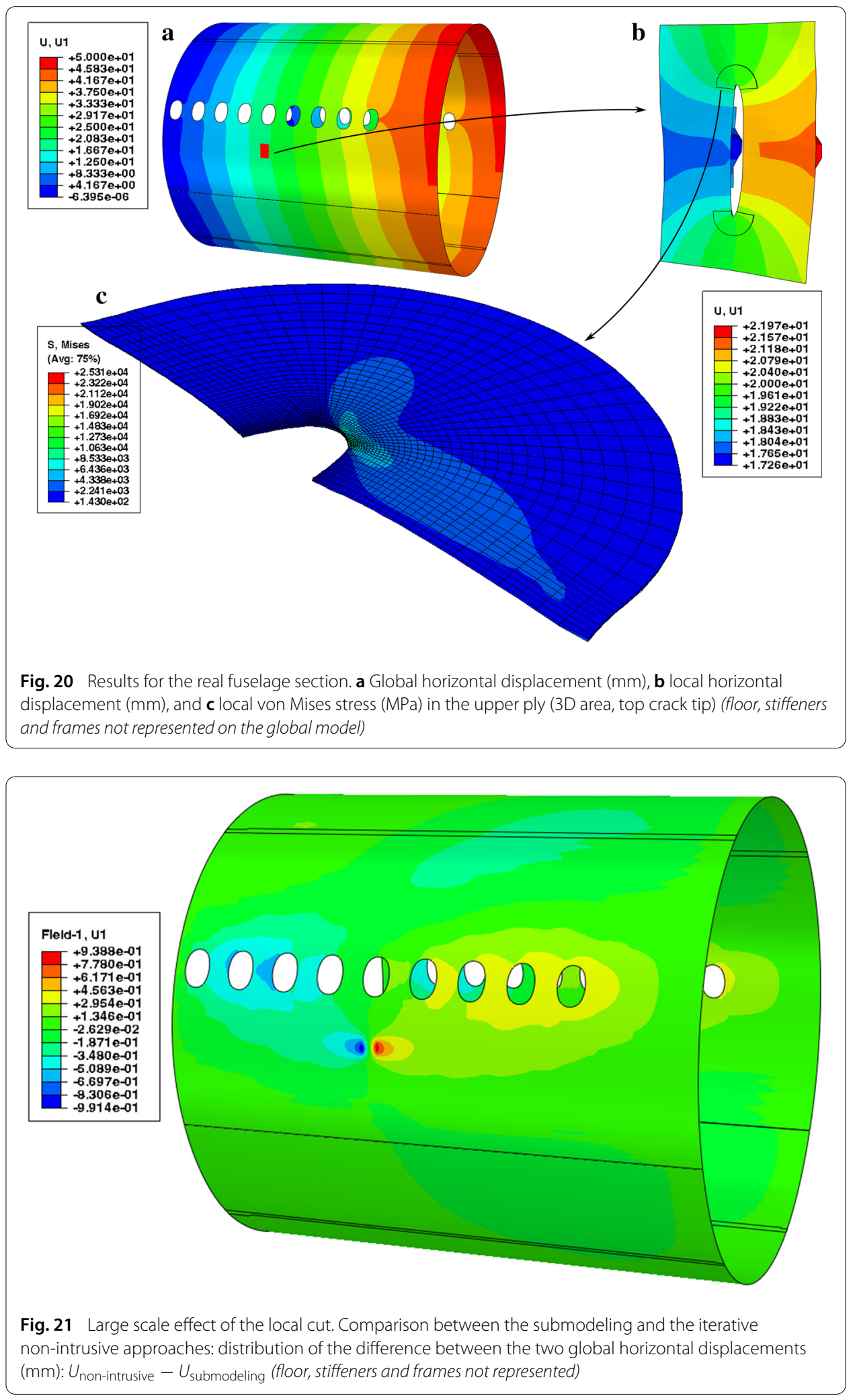
intrusive method, which happens to be physically relevant. As a complement, we compare the results obtained for the global model to the one we get using the classical top-down submodeling approach (see Fig. 21). We clearly see a discrepancy between the two solutions around the local area which highlights again the necessity of an iterative strategy for a proper force redistribution at the global scale.

\section{Conclusion}

In this paper, we developed a non-intrusive coupling methodology for the multiscale analysis of composite structures with elastic shell representation at global scale and solid modeling at local scale. The innovative character of the approach concerns its suitability to actual situations stress engineers are meeting daily: it allows coupling non-matching meshes (different level of refinement of the face-to-face meshes), but also non-conforming geometries (the local finite elements are not aligned with the edges of the coarse elements), and even topologically incompatible models (global shell versus local solid). Indeed, the construction of the local model is now fully relaxed from any meshing constraint emanating from the global model. To accomplish these enhancements, we employed an original and rather pragmatic strategy: it was proposed to expand the initial independent solid local model, by means of the construction of a shell transition mesh, in order to recover the robust situation of a geometrically and topologically conforming global/local interface. For the sake of minimal intrusiveness, the shell/solid coupling is managed through features available in commercial software (shell-to-solid coupling).

In this work, an important step towards the transfer of the approach to the industrial environment was achieved: a demonstrator of the method was developed in Abaqus v6.14 FE code in order to facilitate its dissemination to Airbus Group: a dedicated Abaqus python script automates the preparation of global and local models; native Abaqus v6.14 co-simulation capabilities have been extended through specific developments. Such developments were successfully applied to solve representative use-cases extracted from engineering practices. In particular, the global/local simulation of an aircraft fuselage section subjected to engine burst has been achieved. The characteristic lengths at global and local scales were $7 \mathrm{~m}$ and $10 \mu \mathrm{m}$, respectively, and the global model was about $10 \times 10^{6}$ degrees of freedom. According to our knowledge, this is the first time in the context of nonintrusive coupling that such a realistic industrial structure has been computed. Through the numerical experiments conducted, we were able to highlight the benefits reached from the proposed methodology, which, in our opinion, constitutes a relevant framework to tackle realistic engineering (especially aeronautical) applications:

- No human intervention on the global model is needed the local geometry is not represented at the global scale, which enables to take into account critical situations where the local details have a characteristic length of the same order of magnitude as the global elements size in the area of interest.

- Severe heterogeneity between the two models is well captured stress concentration around the local detail can result in damage within the laminate's sub-constituents, which can be captured through a relevant solid mesh and an appropriate constitutive law, while the global behavior remains modeled by the shell elements and an homogenized elasticity. 
- Significant global effects due to local perturbations are accounted for stress concentration induced by local perturbations involves a strongly non-linear behavior at local scale which is well transmitted to the global scale through the coupling strategy (global displacements are sensitive to damage reached locally).

- Full compatibility with industrial framework is met the analysis process, numerical data flow and modeling path do not involve any additional features when compared to industrial state-of-the-art methodologies (sub-modeling); in particular, the global model is provided as an orphan mesh, which prevents any modification at a global scale: this is a common situation met in stress offices, especially when the construction of meshes is outsourced.

\section{Authors' contributions}

All authors have prepared the manuscript. All authors read and approved the final manuscript.

\section{Author details}

${ }^{1}$ Airbus SAS, Toulouse, France, ${ }^{2}$ IMT-Université de Toulouse, INSA-UPS-CNRS, Toulouse, France, ${ }^{3}$ Instituto Tecnológico de Aeronáutica (ITA), São José dos Campos, Brazil, ${ }^{4}$ ICA-Université de Toulouse, INSA-UPS-Mines Albi-ISAE-CNRS, Toulouse, France.

\section{Acknowledgements}

The authors would like to acknowledge the financial support of the French National Research Agency under Grant ICARE ANR-12-MONU-0002. The third author, Mateus Toniolli, would like also to thank Airbus Group Innovations for hosting him for his master internship during summer 2016.

\section{Competing interests}

The authors declare that they have no competing interests.

Availability of data and materials

Not applicable.

\section{Consent for publication}

Not applicable.

Ethics approval and consent to participate

Not applicable.

Funding

French National Research Agency under Grant ICARE ANR-12-MONU-0002

\section{Publisher's Note}

Springer Nature remains neutral with regard to jurisdictional claims in published maps and institutional affiliations.

Received: 27 November 2017 Accepted: 9 December 2017

Published online: 05 January 2018

References

1. Bhardwaj M, Pierson K, Reese G, Walsh T, Day D, Alvin K, Peery J, Farhat C, Lesoinne M. Salinas: a scalable software for high-performance structural and solid mechanics simulations. In: Proceedings of SC02. New York: ACM; 2002.

2. Schillinger D, Dede L, Scott MA, Evans JA, Borden MJ, Rank E, Hughes TJR. An isogeometric design-through-analysis methodology based on adaptive hierarchical refinement of nurbs, immersed boundary methods, and t-spline cad surfaces. Comput Methods Appl Mech Eng. 2012;249-252:116-50.

3. Schillinger $D$, Ruess $M$. The finite cell method: a review in the context of higher-order structural analysis of cad and image-based geometric models. Arch Comput Methods Eng. 2015;22(3):391-455.

4. Zander N, Bog T, Elhaddad M, Espinoza R, Hu H, Joly A, Wu C, Zerbe P, Duster A, Kollmannsberger S, Parvizian J, Ruess M, Schillinger D, Rank E. Fcmlab: a finite cell research toolbox for matlab. Adv Eng Softw. 2014;74:49-63.

5. Kelley FS. Mesh requirements for the analysis of a stress concentration by the specified boundary displacement method. In: Proceedings of the second international computers in engineering conference. ASME; 1982.

6. Jara-Almonte CC, Knight CE. The specified boundary stiffness/force sbsf method for finite element subregion analysis. Int J Numer Methods Eng. 1988;26(7):1567-78.

7. Srinivasan S, Biggers SB, Latour RA. Identifying global/local interface boundaries using an objective search method. Int J Numer Methods Eng. 1996;39:805-28.

8. Cormier NG, Smallwood BS, Sinclair GB, Meda G. Aggressive submodeling of stress concentrations. Int J Numer Methods Eng. 1999;46(6):889-909. 
9. Cresta P, Allix O, Rey C, Guinard S. Nonlinear localization strategies for domain decomposition methods in structural mechanics. Comput Methods Appl Mech Eng. 2007;196:1436-46.

10. Gendre L, Allix O, Gosselet P. Non-intrusive and exact global/local techniques for structural problems with local plasticity. Comput Mech. 2009;44:233-45.

11. Hirai I, Wang BP, Pilkey WD. An efficient zooming method for finite element analysis. Int J Numer Methods Eng. 1984;20(9):1671-83.

12. Wyart E, Duflot M, Coulon D, Martiny P, Pardoen T, Remacle JF, Lani F. Substructuring fe-xfe approaches applied to three-dimensional crack propagation. J Comput Appl Math. 2008;215(2):626-38.

13. Touzeau J, Chiaruttini V, Feyel F, Dhia HB. Approche multiéchelle arlequin pour l'étude des structures composites stratifiées. In: 10e Colloque National en Calcul des Structures, Giens; 2011.

14. Whitcomb J. Iterative global/local finite element analysis. Comput Struct. 1991;40(4):1027-31.

15. Gupta P, Pereira JP, Kim D-J, Duarte CA, Eason T. Analysis of three-dimensional fracture mechanics problems: a non-intrusive approach using a generalized finite element method. Eng Fract Mech. 2012;90:41-64.

16. Passieux J-C, Réthoré J, Gravouil A, Baietto M-C. Local/global non-intrusive crack propagation simulation using a multigrid X-FEM solver. Comput Mech. 2013;56(2):1381-93.

17. Chevreuil M, Nouy A, Safatly E. A multiscale method with patch for the solution of stochastic partial differential equations with localized uncertainties. Comput Methods Appl Mech Eng. 2013;255:255-74

18. Guguin G, Allix O, Gosselet P, Guinard S. Nonintrusive coupling of $3 \mathrm{D}$ and $2 \mathrm{D}$ laminated composite models based on finite element 3D recovery. Int J Numer Methods Eng. 2014;98(5):324-43.

19. Guguin G, Allix O, Gosselet P, Guinard S. On the computation of plate assemblies using realistic 3d joint model: a non-intrusive approach. Adv Model Simul Eng Sci. 2016;3(1):1-18.

20. Duval M, Passieux J-C, Salaün M, Guinard S. Non-intrusive coupling: recent advances and scalable nonlinear domain decomposition. Arch Comput Methods Eng. 2016;23(1):17-38.

21. Chantrait T, Rannou J, Gravouil A. Low intrusive coupling of implicit and explicit time integration schemes for structural dynamics: application to low energy impacts on composite structures. Finite Elem Anal Design. 2014:86:23-33.

22. Bettinotti $\mathrm{O}$, Allix $\mathrm{O}$, Perego $\mathrm{U}$, Oancea $\mathrm{V}$, Malherbe $\mathrm{B}$. A fast weakly intrusive multiscale method in explicit dynamics. Int J Numer Methods Eng. 2014;100(8):577-95.

23. Fish J, Shek K. Multiscale analysis of large scale nonlinear structures and materials. Int J Comput Civil Struct Eng. 2000;1:79-90.

24. Steger JL. The chimera method of flow simulation. In: Workshop on Applied CFD, Univ of Tennessee Space Institute; 1991.

25. Hughes TJR, Feijoo GR, Mazzei L, Quincy J-B. The variarional multiscale method-a paradigm for computational mechanics. Comput Methods Appl Mech Eng. 1998;166:3-24.

26. Hund A, Ramm E. Locality constraints within multiscale model for non-linear material behaviour. Int J Numer Methods Eng. 2007;70:1613-32.

27. Glowinski R, He J, Lozinski A, Rappaz J, Wagner J. Finite element approximation of multi-scale elliptic problems using patches of elements. Numer Math. 2005;101(4):663-87.

28. Lozinski A, Pironneau O. Numerical zoom for localized multiscales. Numer Methods Partial Diff Equ. 2011:27:197-207.

29. Markovič D, Ibrahimbegović A. On micro-macro interface conditions for micro scale based FEM for inelastic behavior of heterogeneous materials. Comput Methods Appl Mech Eng. 2004;193:5503-23.

30. Rank E. Adaptive remeshing and h-p domain decomposition. Comput Methods Appl Mech Eng. 1992;101:299-313.

31. Duster A, Niggl A, Rank E. Applying the hp-d version of the fem to locally enhance dimensionally reduced models. Comput Methods Appl Mech Eng. 2007;196:3524-33.

32. Bouclier R, Passieux J-C, Salaün M. Local enrichment of nurbs patches using a non-intrusive coupling strategy: geometric details, local refinement, inclusion, fracture. Comput Methods Appl Mech Eng. 2016;300:1-26.

33. Bouclier R, Passieux J-C, Salaün M. Development of a new, more regular, mortar method for the coupling of nurbs subdomains within a nurbs patch: application to a non-intrusive local enrichment of nurbs patches. Comput Methods Appl Mech Eng. 2017;316:123-50.

34. Liu YJ, Sun Q, Fan XL. A non-intrusive global/local algorithm with non-matching interface: derivation and numerical validation. Comput Methods Appl Mech Eng. 2014;277:81-103.

35. Gander M, Japhet C. Algorithm 932: Pang: software for non-matching grid projections in $2 \mathrm{~d}$ and $3 \mathrm{~d}$ with linear complexity. ACM Trans Math Softw Assoc Comput Mach. 2013;40(1):6-1625.

36. Abaqus 6.14 documentation, keywords reference guide. Shell-to-solid coupling.

37. Guinard S. Damage prediction and validation for low velocity impacts (maaximus deliverable d.3.4.3). Technical report. 2014 